\title{
Synthesis, spectral studies, pharmacological and insect antifeedant activities of some (E)-1-(2-(2-benzyloxy-3-methacrylate))-1-naphthyl enones
}

\author{
G. Thirunarayanan ${ }^{*}$, , S. Dineshkumar ${ }^{1}$, R. Arulkumaran ${ }^{2}$, R. Sundararajan ${ }^{2}$, \\ D. Kamalakkannan ${ }^{2}$, R. Suresh ${ }^{2}$, SP. Sakthinathan ${ }^{2}$, V. Manikandan ${ }^{2}$, \\ G. Vanangamudi ${ }^{2}$
}

${ }^{1}$ Department of Chemistry, Annamalai University, Annamalainagar-608 002, Tamilnadu, India.

${ }^{2}$ Post Graduate and Research Department of Chemistry, Government Arts College, C-Mutlur-608 102, Tamilnadu, India.

E-mail address: thirunarayanan.g.10313@annamalaiuniversity.ac.in; drgtnarayanan@mail.com

Keywords: (E)-2-Benzyloxy-(3-methoxy acrylate)-1-naphthyl enones; Hammett equation; Antimicrobial activity; Antioxidant activity; Insect antifeedant activity.

ABSTRACT. Totally twelve title compounds have been synthesized by Claisen-Schmidt condensation of (E)-methyl 2-(2-(((1-acetylnaphthalen-2-yl)oxy)methyl)phenyl)-3-methoxy acrylate and substituted benzaldehydes in presence of catalytic quantity of $\mathrm{LiOH} . \mathrm{H}_{2} \mathrm{O}$ catalyst under stirring in room temperature. The yields of the compounds are more than $92 \%$. The synthesized titled compounds were characterized by their physical constants, IR, NMR and Mass spectral data. The spectral data such as infrared $v \mathrm{CO}_{\text {s-cis, s-trans }}, \mathrm{CO}_{\text {ester }}$, NMR chemical shifts $(\delta, \mathrm{ppm})$ of $\mathrm{H}_{\alpha}, \mathrm{H}_{\beta}$, $\mathrm{C}_{\alpha}, \mathrm{C}_{\beta}, \mathrm{CO}$ of enone moiety, $\mathrm{H}_{\beta^{\prime}}$ and $\mathrm{CO}$ of acrylate moieties have been correlated with Hammett substituent constants, $\mathrm{F}$ and $\mathrm{R}$ parameters. The antimicrobial, antioxidant and insect antifeedant activities of all synthesized compounds have been evaluated using corresponding bacterial and fungal strains, DPPH radical scavenging and Disc diffusion bio-assay of $4^{\text {th }}$ instar larvae Achoea Janata $L$ with castor-leaf discs.

\section{INTRODUCTION}

Currently, chemists and organic synthetic researcher have paid much more attention for green solvent assited and ground chemistry synthesis[1-6]. These synthetic methodology have merits over than conventional methods such as easy work-up procedure, handling technique, nonhazardousness, environmental pollution free from atmosphere, shorter reaction time and better yields[7]. Aryl enones and methacrylates possess many biological activities such as antimicrobial[8,9], acute toxicity[10], haemo toxicity[11], in-vivo toxicity[12], genotoxicity[13], antioxidant[14], anticancer[15], antiviral[16], site-specific peroral delivery[17] antiinflammatory/analgesic[18], anti plasmodial[19], anti-tuberclosis[20] and insect antifeedant[21, 22] activities due to presence of carbonyl and vinyl segments present in their structural moieties. Various catalysts were used for synthesis of enones by conventional heating methods[4, 23, 24, 25]. Spectroscopic data have been utilized for prediction of ground state equilibration of organic molecules such as styrenes, $E$ s-cis and s-trans styryl ketones, unsaturated acids and their derivatives, gauche- and anti- form of $\omega$-halo acyl and its esters[26, 27]. Infrared spectroscopy is one of the tool for studying structural elucidation, qualitative and quantitative analysis. NMR spectroscopy is useful for prediction of spatial arrangement of protons such as cis-and trans- refer with coupling constants and their interactions[28]. Spectroscopic data is used for studying structureactivity relationships of organic substrates through Hammett equation. Recently John Joseph et. al., have synthesized and studied the spectral correlation and antimicrobial activities of some methoxy substituted phenyl chalcones ${ }^{[29]}$. Thirunarayanan and his co-workers have studied the spectral correlation and pharmacological evaluation of chalcones, ${ }^{1} H$ pyrazolines, $\mathrm{N}$-acetyl- ${ }^{1} H$-Pyrazolines, aryl acyl bromides and aryl $\omega$-halo esters[27, 30, 31]. Sundararajan et al., have studied the solventfree synthesis, spectral QSAR study antimicrobial and insect antifeedant activities of poly halo 
substituted phenyl chalcones [32]. Within the above view, there is no report available for the synthesis, spectral studies and biological evaluation of (E)-2-benzyloxy-(3-methoxy acrylate)-1naphthyl enones. Therefore the author have taken effort to synthesis and studied the spectral and biological evaluation such as antimicrobial, antioxidant and insect antifeedant activities of the title compound.

\section{EXPERIMENTAL}

\subsection{General}

All chemicals used in the present investigation were procured from Sigma-Aldrich and E-Merck chemical company. Melting points of all chalcones were determined in open glass capillaries on Mettler FP51 melting point apparatus and are uncorrected. Infrared spectra (KBr, $4000-400 \mathrm{~cm}^{-1}$ ) were recorded on Thermo Nicolet iS5 Fourier transform spectrophotometer. he NMR spectra of synthesized compounds were recorded in BRUKER AV 400 NMR spectrometer operating at $400 \mathrm{MHz}$ was used for ${ }^{1} \mathrm{H}$ spectra and $100 \mathrm{MHz}$ for ${ }^{13} \mathrm{C}$ spectra in $\mathrm{CDCl}_{3}$ solvent using TMS as internal standard. Mass spectra were recorded on a SIMADZU GC-MS2010 Spectrometer using Electron Impact (EI) techniques.

\subsection{Synthesis of 2-Benzyloxy-(3-methacylate)-1-acetyl naphthalene}

Equimolar quantities of 2-hydroxy-1-acetylnaphthalene (1 mmol), 2-(3-methacylate)-benzyl bromide $0.5 \mathrm{~g}$ of sodium hydroxide and $20 \mathrm{~mL}$ were vigorously stirred in magnetic stirrer at room temperature for $6 \mathrm{~h}$. The completion of reaction was monitored by TLC. After completion of the reaction, the reaction mixtures was treated with $20 \mathrm{~mL}$ of $0.2 \mathrm{~N} \mathrm{HCl}$ and thoroughly washed with water, the obtained solid was filtered. The crude compound was crystallized with ethanol.

\subsection{General procedure for synthesis of $(E)-1-(2-(2-b e n z y l o x y-(3-m e t h o x y a c r y l a t e))-1-n a p h t h y l$} enones

An appropriate equi molar mixture of 2-benzyloxy-(3-methacylate)-1-acetyl naphthalene (2 $\mathrm{mmol})$ in $5 \mathrm{~mL}$ of ethanol and $\mathrm{LiOH} . \mathrm{H}_{2} \mathrm{O}(8 \mathrm{mg}, 0.2 \mathrm{mmol})$ were stirred in magnetic stirrer at room temperature. To this substituted benzaldehydes $(2 \mathrm{mmol})$ were added and the stirring was continued for 35-40 mins. The completion of the reaction was monitored by TLC. After completion of the reaction, the ethanol was removed by reduced pressure distillation. The residue obtained was treated with $10 \mathrm{~mL}$ of water, neutralized with diluted $\mathrm{HCl}(15 \mathrm{~mL}, 2 \%)$ and extracted with $20 \mathrm{~mL}$ of ethyl acetate. The collective extract was washed with $10 \mathrm{~mL}$ of brine solution and dried over on sodium sulphate. Evaporation of solvent gave the crude chalcones. It is further purified by column chromatogram using $\mathrm{CH}_{2} \mathrm{Cl}_{2}$ :EtOAc $(1: 3)$ as eluents afforded more than $88 \%$ yield as pale yellow glittering solids. The physical constants and mass fragments $(\mathrm{m} / \mathrm{z})$ of the chalcones have been presented in Table 1.

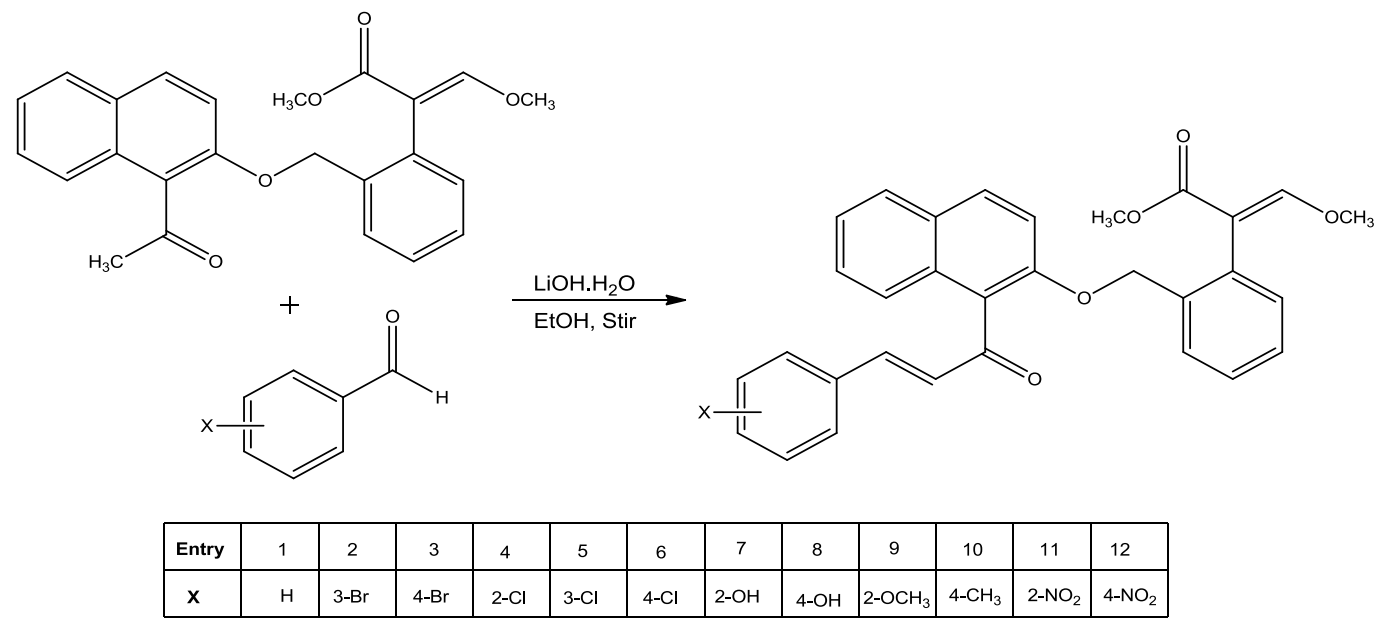

Scheme 1. Synthesis of (E)-2-benzyloxy-(3-methoxyacrylate)-1-naphthyl enones 


\section{RESULTS AND DISCUSSION}

\subsection{Infrared spectral study}

In the present investigation, the authors have studied the effect of substituents on the infrared carbonyl stretches $\left(\mathrm{cm}^{-1}\right)$ of keto- and ester- moiety of the synthesized (E)-2-benzyloxy-(3methoxyacrylate)-1-naphthyl enones. The $\mathrm{CO}_{\text {s-cis }}$ and ${ }_{\text {s-trans }}$ conformers are shown in Fig. 1. And the corresponding stretches of keto- and ester- moiety of the synthesized (E)-1-(2-(2-benzyloxy-(3methoxyacrylate))-1-naphthyl enones were presented in Table 2. These stretches were correlated with Hammett substituent constants, $\mathrm{F}$ and $\mathrm{R}$ parameters using single and multi-linear regression analysis[2, 6,14,19, 24-32]. In the infrared spectral correlation, the Hammett equation is employed as in the form of

$$
v=\rho \sigma+v_{0}
$$

where $v$ is the frequency for the members of the series; $\rho$ is the reaction constant; $\sigma$ is the substituent constant and $v_{\mathrm{o}}$ is the frequency for the parent member of the series.

Table 1. Physical constants, analytical and mass fragments of (E)1-(2-(2-benzyloxy-(3methoxyacrylate)))-1-naphthylenones

\begin{tabular}{|l|l|l|l|l|l|l|}
\hline Entry & $\mathbf{X}$ & $\mathbf{M . F}$. & $\mathbf{M . W}$ & $\begin{array}{l}\text { Yield } \\
(\mathbf{\%})\end{array}$ & $\begin{array}{l}\mathbf{m . p .} \\
(\cdot \mathbf{C})\end{array}$ & Mass(m/z) \\
\hline $\mathbf{1}$ & $\mathrm{H}$ & $\mathrm{C}_{31} \mathrm{H}_{26} \mathrm{O}_{5}$ & 478 & 92 & $117-118$ & $478\left[\mathrm{M}^{+}\right], 347,363,279,205,131,115,77,15$ \\
\hline $\mathbf{2}$ & $3-\mathrm{Br}$ & $\mathrm{C}_{31} \mathrm{H}_{25} \mathrm{BrO}_{5}$ & 557 & 90 & $102-103$ & $\begin{array}{l}557\left[\mathrm{M}^{+}\right], 558\left[\mathrm{M}^{2+}\right], 441,351,375,205,180,115,90, \\
79,77,15\end{array}$ \\
\hline $\mathbf{3}$ & $4-\mathrm{Br}$ & $\mathrm{C}_{31} \mathrm{H}_{25} \mathrm{BrO}_{5}$ & 557 & 91 & $114-115$ & $557\left[\mathrm{M}^{+}\right], 558\left[\mathrm{M}^{2+}\right], 388,375,335,221,180,167,79,77$ \\
\hline $\mathbf{4}$ & $2-\mathrm{Cl}$ & $\mathrm{C}_{31} \mathrm{H}_{25} \mathrm{ClO}_{5}$ & 512 & 90 & $123-124$ & $512\left[\mathrm{M}^{+}\right], 514\left[\mathrm{M}^{2+}\right], 453,291,375,137,77,59,35$ \\
\hline $\mathbf{5}$ & $3-\mathrm{Cl}$ & $\mathrm{C}_{31} \mathrm{H}_{25} \mathrm{ClO}_{5}$ & 512 & 90 & $136-137$ & $\begin{array}{l}512\left[\mathrm{M}^{+}\right], 514\left[\mathrm{M}^{2+}\right], 453,321,291,221,191,126,124, \\
77,35\end{array}$ \\
\hline $\mathbf{6}$ & $4-\mathrm{Cl}$ & $\mathrm{C}_{31} \mathrm{H}_{25} \mathrm{ClO}_{5}$ & 512 & 91 & $119-120$ & $512\left[\mathrm{M}^{+}\right], 514\left[\mathrm{M}^{2+}\right], 401,397,307,205,115,111,77,35$ \\
\hline $\mathbf{7}$ & $2-\mathrm{OH}$ & $\mathrm{C}_{31} \mathrm{H}_{26} \mathrm{O}_{6}$ & 494 & 92 & $98-99$ & $494,375,347,289,205,147,119,77,17$ \\
\hline $\mathbf{8}$ & $4-\mathrm{OH}$ & $\mathrm{C}_{31} \mathrm{H}_{26} \mathrm{O}_{6}$ & 494 & 92 & $107-108$ & $494,388,303,273,221,191,106,77,17$ \\
\hline $\mathbf{9}$ & $2-$ & $\mathrm{C}_{32} \mathrm{H}_{28} \mathrm{O}_{6}$ & 508 & 93 & $124-125$ & $\begin{array}{l}508,393,375,347,287,221,161,133,115,91,77, \\
31,15\end{array}$ \\
\hline $\mathbf{1 0}$ & $4-\mathrm{CH}_{3}$ & $\mathrm{C}_{32} \mathrm{H}_{28} \mathrm{O}_{5}$ & 492 & 93 & $115-116$ & $492,401,347,377,145,115,91,77,15$ \\
\hline $\mathbf{1 1}$ & $2-\mathrm{NO}_{2}$ & $\mathrm{C}_{31} \mathrm{H}_{25} \mathrm{NO}_{7}$ & 523 & 90 & $127-128$ & $523,388,347,302,221,176,135,77,59$ \\
\hline $\mathbf{1 2}$ & $4-\mathrm{NO}_{2}$ & $\mathrm{C}_{31} \mathrm{H}_{25} \mathrm{NO}_{7}$ & 523 & 90 & $134-135$ & $523,408,375,318,205,148,115,77,59$ \\
\hline
\end{tabular}

Table 2. The infrared $v \mathrm{CO}_{s-c i s}$, s-trans, $\mathrm{CO}_{\text {ester, }}$, stretches $\left(\mathrm{cm}^{-1}\right)$, NMR chemical shifts $(\delta, \mathrm{ppm})$ of $\mathrm{H}_{\alpha}$, $\mathrm{H}_{\beta}, \mathrm{H}_{\beta^{\prime}}, \mathrm{C}_{\alpha}, \mathrm{C}_{\beta}, \mathrm{CO}_{\mathrm{ester}}, \mathrm{C}_{\alpha^{\prime}}, \mathrm{C}_{\beta^{\prime}}$, of (E)-1-(2-benzyloxy-(3-methoxyacrylate))-1-naphthyl enones

\begin{tabular}{|c|c|c|c|c|c|c|c|c|c|c|c|c|c|}
\hline \multirow[t]{2}{*}{ Entry } & \multirow[t]{2}{*}{$\mathbf{X}$} & \multicolumn{3}{|l|}{ IR } & \multicolumn{3}{|c|}{${ }^{1}$ H NMR } & \multicolumn{6}{|c|}{${ }^{13} \mathrm{C}$ NMR } \\
\hline & & $\begin{array}{c}\mathrm{CO} \\
s-c i s\end{array}$ & $\begin{array}{l}\text { CO } \\
\text { s-trans }\end{array}$ & $\mathrm{CO}_{\text {ester }}$ & $\mathbf{H}_{\boldsymbol{\alpha}}$ & $\mathbf{H}_{\beta}$ & $\mathbf{H}_{\boldsymbol{\beta}^{\prime}}$ & $\mathrm{CO}$ & $\overline{C_{\alpha}}$ & $\overline{C_{\beta}}$ & $\mathrm{CO}_{\text {ester }}$ & $\mathbf{C}_{\boldsymbol{a}^{\prime}}$ & $\mathbf{C}_{\boldsymbol{\beta}^{\prime}}$ \\
\hline 1 & $\mathrm{H}$ & 1673 & 1659 & 1603 & 7.386 & 7.916 & 8.314 & 189.13 & 123.57 & 139.62 & 164.81 & 114.74 & 149.38 \\
\hline 2 & $3-\mathrm{Br}$ & 1676 & 1641 & 1610 & 7.324 & 7.924 & 8.004 & 189.97 & 123.56 & 139.74 & 164.25 & 117.46 & 149.72 \\
\hline 3 & $4-\mathrm{Br}$ & 1674 & 1648 & 1605 & 7.431 & 7.859 & 8.101 & 190.32 & 124.16 & 141.36 & 162.35 & 115.45 & 151.63 \\
\hline 4 & $2-\mathrm{Cl}$ & 1678 & 1634 & 1607 & 7.513 & 7.267 & 8.141 & 190.64 & 124.25 & 140.77 & 165.26 & 117.12 & 152.12 \\
\hline 5 & $3-\mathrm{Cl}$ & 1680 & 1647 & 1603 & 7.501 & 7.358 & 8.091 & 189.71 & 124.23 & 138.74 & 164.59 & 112.81 & 153.72 \\
\hline 6 & 4-Cl & 1676 & 1643 & 1618 & 7.361 & 7.311 & 8.172 & 189.47 & 124.93 & 139.87 & 165.29 & 112.70 & 151.92 \\
\hline 7 & $2-\mathrm{OH}$ & 1669 & 1635 & 1603 & 7.205 & 7.671 & 8.095 & 187.76 & 123.02 & 137.34 & 166.89 & 108.99 & 149.85 \\
\hline 8 & $4-\mathrm{OH}$ & 1672 & 1640 & 1614 & 7.311 & 7.809 & 8.112 & 189.17 & 124.17 & 139.33 & 164.54 & 109.24 & 150.07 \\
\hline 9 & $\begin{array}{l}2- \\
\mathrm{OCH}_{3}\end{array}$ & 1664 & 1638 & 1597 & 7.017 & 7.717 & 7.904 & 184.37 & 124.03 & 138.74 & 163.25 & 107.58 & 148.26 \\
\hline 10 & $4-\mathrm{CH}_{3}$ & 1666 & 1641 & 1604 & 7.321 & 7.964 & 8.119 & 183.46 & 124.01 & 139.66 & 163.58 & 108.36 & 148.58 \\
\hline 11 & $2-\mathrm{NO}_{2}$ & 1683 & 1658 & 1610 & 7.734 & 8.316 & 8.410 & 191.75 & 125.26 & 141.65 & 165.56 & 118.59 & 151.66 \\
\hline 12 & $4-\mathrm{NO}_{2}$ & 1686 & 1665 & 1621 & 7.774 & 8.567 & 8.560 & 191.96 & 125.69 & 142.01 & 165.95 & 118.85 & 151.92 \\
\hline
\end{tabular}


<smiles>[Y][I-]c1ccc(/C=C/C(=O)c2c(OCc3ccccc3/C(=C\OC)C(=O)OC)ccc3ccccc23)cc1</smiles>

Fig. 1. The s-cis and s-trans conformers of (E)-2-benzyloxy-(3-methoxyacrylate)-1-naphthyl enones

The results of statistical analysis are shown in Table 3. From the Table 3, The $\mathrm{CO}_{\text {s-cis }}$ stretches of enones correlated satisfactorily with Hammett substituent constants, F and R parameters. The $\mathrm{CO}_{\text {s-trans }}$ stretches of enones correlated satisfactorily with Hammett $\sigma, \sigma^{+}, \sigma_{\mathrm{I}}$, constants and $\mathrm{R}$ parameters. The Hammett $\sigma_{\mathrm{R}}$ constant and $\mathrm{F}$ parameter were fail in correlation. The $\mathrm{CO}_{\text {ester }}$ stretches of enones correlated satisfactorily with Hammett substituent constants and $\mathrm{F}$ parameter. The $\mathrm{R}$ parameter was fail in correlation. All correlation gave positive $\rho$ values. This implies that the normal substituent effect operates in all system. The degree of transmission of substituent effect on the $v \mathrm{CO}_{\text {s-cis }}$ conformer is higher than that of $v \mathrm{CO}_{\text {s-trans }}$ conformers. The failure in correlation was due to the inability of predicting the effect of substituent on the carbonyl group and associated with the resonance-conjugative structure as shown in Fig. 2.<smiles>CO/C=C(/C(=O)OC)c1ccccc1COc1ccc2ccccc2c1/C([O-])=C\C=C1\C=CC=CC1=[O+]C</smiles>

Fig. 2. The resonance-conjugative structure 
Table 3. Results of statistical analysis of infrared $\mathrm{vCO}_{\text {s-cis }}$, s-trans, $\mathrm{CO}_{\text {ester, }}$ stretches $\left(\mathrm{cm}^{-1}\right)$ of 1-(2benzyloxy-(3-methoxyacrylate))-1-naphthyl enones with Hammett $\sigma, \sigma^{+}, \sigma_{\mathrm{I}}, \sigma_{\mathrm{R}}$, constants, F and R parameters.

\begin{tabular}{|c|c|c|c|c|c|c|c|}
\hline Frequency & Constant & $\mathrm{r}$ & I & $\rho$ & $\mathrm{s}$ & $\mathrm{n}$ & Correlated derivatives \\
\hline \multirow[t]{6}{*}{$\mathrm{CO}_{s-c i s}$} & $\sigma$ & 0.924 & 1672.43 & 15.495 & 2.62 & 12 & $\begin{array}{l}\mathrm{H}, 3-\mathrm{Br}, 4-\mathrm{Br}, 2-\mathrm{Cl}, 3-\mathrm{Cl}, 4-\mathrm{Cl}, 2-\mathrm{OH}, 4-\mathrm{OH}, \\
2-\mathrm{OCH}_{3}, 4-\mathrm{CH}_{3}, 2-\mathrm{NO}_{2}, 3-\mathrm{NO}_{2}\end{array}$ \\
\hline & $\sigma^{+}$ & 0.912 & 1673.72 & 10.260 & 3.82 & 12 & $\begin{array}{l}\mathrm{H}, 3-\mathrm{Br}, 4-\mathrm{Br}, 2-\mathrm{Cl}, 3-\mathrm{Cl}, 4-\mathrm{Cl}, 2-\mathrm{OH}, 4-\mathrm{OH}, \\
2-\mathrm{OCH}_{3}, 4-\mathrm{CH}_{3}, 2-\mathrm{NO}_{2}, 3-\mathrm{NO}_{2}\end{array}$ \\
\hline & $\sigma_{\mathrm{I}}$ & 0.907 & 1664.30 & 23.032 & 4.31 & 12 & $\begin{array}{l}\mathrm{H}, 3-\mathrm{Br}, 4-\mathrm{Br}, 2-\mathrm{Cl}, 3-\mathrm{Cl}, 4-\mathrm{Cl}, 2-\mathrm{OH}, 4-\mathrm{OH}, \\
2-\mathrm{OCH}_{3}, 4-\mathrm{CH}_{3}, 2-\mathrm{NO}_{2}, 3-\mathrm{NO}_{2}\end{array}$ \\
\hline & $\sigma_{\mathrm{R}}$ & 0.917 & 1678.47 & 18.834 & 4.02 & 12 & $\begin{array}{l}\mathrm{H}, 3-\mathrm{Br}, 4-\mathrm{Br}, 2-\mathrm{Cl}, 3-\mathrm{Cl}, 4-\mathrm{Cl}, 2-\mathrm{OH}, 4-\mathrm{OH}, \\
2-\mathrm{OCH}_{3}, 4-\mathrm{CH}_{3}, 2-\mathrm{NO}_{2}, 3-\mathrm{NO}_{2}\end{array}$ \\
\hline & $\mathrm{F}$ & 0.905 & 1665.82 & 21.240 & 4.10 & 12 & $\begin{array}{l}\mathrm{H}, 3-\mathrm{Br}, 4-\mathrm{Br}, 2-\mathrm{Cl}, 3-\mathrm{Cl}, 4-\mathrm{Cl}, 2-\mathrm{OH}, 4-\mathrm{OH}, \\
2-\mathrm{OCH}_{3}, 4-\mathrm{CH}_{3}, 2-\mathrm{NO}_{2}, 3-\mathrm{NO}_{2}\end{array}$ \\
\hline & $\mathrm{R}$ & 0.906 & 1678.69 & 16.146 & 4.22 & 12 & $\begin{array}{l}\mathrm{H}, 3-\mathrm{Br}, 4-\mathrm{Br}, 2-\mathrm{Cl}, 3-\mathrm{Cl}, 4-\mathrm{Cl}, 2-\mathrm{OH}, 4-\mathrm{OH}, \\
2-\mathrm{OCH}_{3}, 4-\mathrm{CH}_{3}, 2-\mathrm{NO}_{2}, 3-\mathrm{NO}_{2}\end{array}$ \\
\hline \multirow[t]{6}{*}{$\mathrm{CO}_{\text {s-trans }}$} & $\sigma$ & 0.906 & 1643.21 & 17.015 & 7.84 & 12 & $\begin{array}{l}\mathrm{H}, 3-\mathrm{Br}, 4-\mathrm{Br}, 2-\mathrm{Cl}, 3-\mathrm{Cl}, 4-\mathrm{Cl}, 2-\mathrm{OH}, 4-\mathrm{OH}, \\
\text { 2- } \mathrm{OCH}_{3}, 4-\mathrm{CH}_{3}, 2-\mathrm{NO}_{2}, 3-\mathrm{NO}_{2}\end{array}$ \\
\hline & $\sigma^{+}$ & 0.905 & 1644.68 & 10.976 & 8.67 & 12 & $\begin{array}{l}\mathrm{H}, 3-\mathrm{Br}, 4-\mathrm{Br}, 2-\mathrm{Cl}, 3-\mathrm{Cl}, 4-\mathrm{Cl}, 2-\mathrm{OH}, 4-\mathrm{OH}, \\
2-\mathrm{OCH}_{3}, 4-\mathrm{CH}_{3}, 2-\mathrm{NO}_{2}, 3-\mathrm{NO}_{2}\end{array}$ \\
\hline & $\sigma_{\mathrm{I}}$ & 0.827 & 1641.11 & 12.637 & 9.07 & 12 & $\begin{array}{l}\mathrm{H}, 3-\mathrm{Br}, 4-\mathrm{Br}, 2-\mathrm{Cl}, 3-\mathrm{Cl}, 4-\mathrm{Cl}, 2-\mathrm{OH}, 4-\mathrm{OH}, \\
2-\mathrm{OCH}_{3}, 4-\mathrm{CH}_{3}, 2-\mathrm{NO}_{2}, 3-\mathrm{NO}_{2}\end{array}$ \\
\hline & $\sigma_{\mathrm{R}}$ & 0.918 & 1652.24 & 12.872 & 5.99 & 12 & $\begin{array}{l}\mathrm{H}, 3-\mathrm{Br}, 4-\mathrm{Br}, 2-\mathrm{Cl}, 3-\mathrm{Cl}, 4-\mathrm{Cl}, 2-\mathrm{OH}, 4-\mathrm{OH}, \\
2-\mathrm{OCH}_{3}, 4-\mathrm{CH}_{3}, 2-\mathrm{NO}_{2}, 3-\mathrm{NO}_{2}\end{array}$ \\
\hline & $\mathrm{F}$ & 0.827 & 1640.38 & 13.339 & 9.10 & 12 & $\begin{array}{l}\mathrm{H}, 3-\mathrm{Br}, 4-\mathrm{Br}, 2-\mathrm{Cl}, 3-\mathrm{Cl}, 4-\mathrm{Cl}, 2-\mathrm{OH}, 4-\mathrm{OH}, \\
2-\mathrm{OCH}_{3}, 4-\mathrm{CH}_{3}, 2-\mathrm{NO}_{2}, 3-\mathrm{NO}_{2}\end{array}$ \\
\hline & $\mathrm{R}$ & 0.907 & 1652.62 & 28.143 & 6.65 & 12 & $\begin{array}{l}\mathrm{H}, 3-\mathrm{Br}, 4-\mathrm{Br}, 2-\mathrm{Cl}, 3-\mathrm{Cl}, 4-\mathrm{Cl}, 2-\mathrm{OH}, 4-\mathrm{OH}, \\
2-\mathrm{OCH}_{3}, 4-\mathrm{CH}_{3}, 2-\mathrm{NO}_{2}, 3-\mathrm{NO}_{2}\end{array}$ \\
\hline \multirow[t]{6}{*}{$\mathrm{CO}_{\text {ester }}$} & $\sigma$ & 0.953 & 1606.49 & 9.543 & 6.17 & 12 & $\begin{array}{l}\mathrm{H}, 3-\mathrm{Br}, 4-\mathrm{Br}, 2-\mathrm{Cl}, 3-\mathrm{Cl}, 4-\mathrm{Cl}, 2-\mathrm{OH}, 4-\mathrm{OH}, \\
2-\mathrm{OCH}_{3}, 4-\mathrm{CH}_{3}, 2-\mathrm{NO}_{2}, 3-\mathrm{NO}_{2}\end{array}$ \\
\hline & $\sigma^{+}$ & 0.891 & 1607.49 & 4.233 & 6.92 & 12 & $\begin{array}{l}\mathrm{H}, 3-\mathrm{Br}, 4-\mathrm{Br}, 2-\mathrm{Cl}, 3-\mathrm{Cl}, 4-\mathrm{Cl}, 2-\mathrm{OH}, 4-\mathrm{OH}, \\
2-\mathrm{OCH}_{3}, 4-\mathrm{CH}_{3}, 2-\mathrm{NO}_{2}, 3-\mathrm{NO}_{2}\end{array}$ \\
\hline & $\sigma_{\mathrm{I}}$ & 0.915 & 1601.96 & 16.223 & 6.26 & 12 & $\begin{array}{l}\mathrm{H}, 3-\mathrm{Br}, 4-\mathrm{Br}, 2-\mathrm{Cl}, 3-\mathrm{Cl}, 4-\mathrm{Cl}, 2-\mathrm{OH}, 4-\mathrm{OH}, \\
2-\mathrm{OCH}_{3}, 4-\mathrm{CH}_{3}, 2-\mathrm{NO}_{2}, 3-\mathrm{NO}_{2}\end{array}$ \\
\hline & $\sigma_{\mathrm{R}}$ & 0.904 & 1610.48 & 13.005 & 6.46 & 12 & $\begin{array}{l}\mathrm{H}, 3-\mathrm{Br}, 4-\mathrm{Br}, 2-\mathrm{Cl}, 3-\mathrm{Cl}, 4-\mathrm{Cl}, 2-\mathrm{OH}, 4-\mathrm{OH}, \\
2-\mathrm{OCH}_{3}, 4-\mathrm{CH}_{3}, 2-\mathrm{NO}_{2}, 3-\mathrm{NO}_{2}\end{array}$ \\
\hline & $\mathrm{F}$ & 0.925 & 1601.05 & 18.642 & 6.14 & 12 & $\begin{array}{l}\mathrm{H}, 3-\mathrm{Br}, 4-\mathrm{Br}, 2-\mathrm{Cl}, 3-\mathrm{Cl}, 4-\mathrm{Cl}, 2-\mathrm{OH}, 4-\mathrm{OH}, \\
2-\mathrm{OCH}_{3}, 4-\mathrm{CH}_{3}, 2-\mathrm{NO}_{2}, 3-\mathrm{NO}_{2}\end{array}$ \\
\hline & $\mathrm{R}$ & 0.734 & 1609.99 & 8.525 & 6.86 & 12 & $\begin{array}{l}\mathrm{H}, 3-\mathrm{Br}, 4-\mathrm{Br}, 2-\mathrm{Cl}, 3-\mathrm{Cl}, 4-\mathrm{Cl}, 2-\mathrm{OH}, 4-\mathrm{OH}, \\
2-\mathrm{OCH}_{3}, 4-\mathrm{CH}_{3}, 2-\mathrm{NO}_{2}, 3-\mathrm{NO}_{2}\end{array}$ \\
\hline
\end{tabular}

$\mathrm{r}=$ correlation coefficient; $\mathrm{I}=$ intercept; $\rho=$ slope; $\mathrm{s}=$ number of correlated derivatives

The inability of some of the single parameter correlation to predict the substituent effects on the carbonyl stretches of the enones, they are worthful when seeking these in multi-correlation with $\sigma_{\mathrm{I}}$ and $\sigma_{\mathrm{R}}$ or $\mathrm{F}$ and R Swain-Lupton's[33] parameters. The generated multi-regression analysis equations are given in (2-7).

$$
\begin{aligned}
& v \mathrm{CO}_{s-c i s}\left(\mathrm{~cm}^{-1}\right)=1670.83( \pm 2.031)+17.993( \pm 4.085) \sigma_{\mathrm{I}}+13.606( \pm 3.626) \sigma_{\mathrm{R}} \\
& (R=0.992, \mathrm{n}=12, P>95 \%) \\
& v \mathrm{CO}_{\text {s-cis }}\left(\mathrm{cm}^{-1}\right)=1670.57( \pm 2.286)+19.672( \pm 4.699) \mathrm{F}+12.335( \pm 3.398) \mathrm{R} \\
& (R=0.990, \mathrm{n}=12, P>95 \%) \\
& v \mathrm{CO}_{\text {s-trans }}\left(\mathrm{cm}^{-1}\right)=1652.01( \pm 4.521)+5.530( \pm 0.091) \sigma_{\mathrm{I}}+32.718( \pm 8.066) \sigma_{\mathrm{R}} \\
& (R=0.982, \mathrm{n}=12, P>95 \%)
\end{aligned}
$$




$$
\begin{aligned}
& v \mathrm{CO}_{\text {s-trans }}\left(\mathrm{cm}^{-1}\right)=1651.30( \pm 5.076)+3.144( \pm 1.014) \mathrm{F}+27.530( \pm 7.549) \mathrm{R} \\
& (R=0.979, \mathrm{n}=12, P>95 \%) \\
& v \mathrm{CO}_{\text {ester }}\left(\mathrm{cm}^{-1}\right)=1605.06( \pm 4.402)+12.786( \pm 8.854) \sigma_{\mathrm{I}}+9.294( \pm 7.841) \sigma_{\mathrm{R}} \\
& (R=0.960, \mathrm{n}=12, P>95 \%) \\
& v \mathrm{VO}_{\text {ester }}\left(\mathrm{cm}^{-1}\right)=1603.06( \pm 4.7101)+16.682( \pm 9.681) \mathrm{F}+5.294( \pm 2.701) \mathrm{R} \\
& (R=0.958, \mathrm{n}=12, P>95 \%)
\end{aligned}
$$

\subsection{NMR spectral study}

\subsection{1. ${ }^{1}$ H NMR spectral study}

In nuclear magnetic resonance spectra, the chemical shifts $(\delta)(\mathrm{ppm})$ of ${ }^{1} \mathrm{H}$ or the ${ }^{13} \mathrm{C}$ nuclei depend on the electronic environment of the nuclei concerned. These chemical shifts have been correlated with reactivity parameters. Thus the Hammett equation was used in the form as shown in (8).

$$
\log \delta=\rho \sigma+\log \delta_{0}
$$

Where $\delta$ is the chemical shift of member of the series; $\rho$ is the reaction constants; $\sigma$ is the substituent constant and $\delta_{0}$ is the chemical shift of the corresponding parent compound of the series. The assigned ${ }^{1} \mathrm{H}$ NMR chemical shifts $(\delta, \mathrm{ppm})$ of $\mathrm{H}_{\alpha}, \mathrm{H}_{\beta}$ of enone and $\mathrm{H}_{\beta^{\prime}}$ protons of enone and acrylate moieties of 2-benzyloxy-(3-methoxyacrylate)-1-naphthyl enones are presented in Table 2 . These chemical shifts $(\mathrm{ppm})$ were correlated with Hammett substituent constants, F and R parameters using single and multi-regression analysis[2, 6,14,19, 24-32]. The results of statistical analyses are given in Table 4. From the Table 4, the correlation of $\delta \mathrm{H}_{\alpha}$ chemical shifts (ppm) of the enones correlated satisfactorily with Hammett substituent constants, $\mathrm{F}$ and $\mathrm{R}$ parameters. The $\delta \mathrm{H}_{\beta}$ and $\delta \mathrm{H}_{\beta^{\prime}}$ chemical shifts (ppm) correlated satisfactorily with Hammett $\sigma, \sigma^{+}, \sigma_{\mathrm{R}}$ constants and $\mathrm{R}$ parameters.

The Hammett $\sigma_{\mathrm{I}}$ constant and $\mathrm{F}$ parameters were fail in the correlations for $\delta \mathrm{H}_{\beta}$ and $\delta \mathrm{H}_{\beta^{\prime}}$ chemical shifts (ppm). The reflectance of substituent effect on the $\delta \mathrm{H}_{\alpha}(\mathrm{ppm})$ is sensitive than $\delta \mathrm{H}_{\beta}$ and $\delta \mathrm{H}_{\beta^{\prime}}$ chemical shifts (ppm). All correlations gave positive $\rho$ values. This shows that that normal substituent effect operates in all systems. The failure in correlation is due to the reason stated earlier and associated with the resonance-conjugative structure as shown in Fig. 2. Some of the single parameter correlations were fail for the $\delta \mathrm{H}_{\beta}$ and $\delta \mathrm{H}_{\beta^{\prime}}$ proton chemical shifts (ppm) with Hammett substituent constants, $\mathrm{F}$ and $\mathrm{R}$ 
Table 4. Results of statistical analysis of ${ }^{1} \mathrm{H}$ NMR chemical shifts $\left(\delta\right.$, ppm) of $\mathrm{H}_{\alpha}, \mathrm{H}_{\beta}$, $\mathrm{H}_{\beta^{\prime}}$ of 1-(2benzyloxy-(3-methoxyacrylate))-1-naphthyl enones with Hammett $\sigma, \sigma^{+}, \sigma_{\mathrm{I}}, \sigma_{\mathrm{R}}$, constants, $\mathrm{F}$ and $\mathrm{R}$ parameters.

\begin{tabular}{|c|c|c|c|c|c|c|c|}
\hline Frequency & Constant & $\mathrm{r}$ & I & $\rho$ & $\mathrm{s}$ & $\mathrm{n}$ & Correlated derivatives \\
\hline \multirow[t]{6}{*}{$\mathrm{H}_{\alpha}$} & $\sigma$ & 0.988 & 7.335 & 0.472 & 0.10 & 12 & $\begin{array}{l}\mathrm{H}, 3-\mathrm{Br}, 4-\mathrm{Br}, 2-\mathrm{Cl}, 3-\mathrm{Cl}, 4-\mathrm{Cl}, 2-\mathrm{OH}, 4-\mathrm{OH}, \\
2-\mathrm{OCH}_{3}, 4-\mathrm{CH}_{3}, 2-\mathrm{NO}_{2}, 3-\mathrm{NO}_{2}\end{array}$ \\
\hline & $\sigma^{+}$ & 0.979 & 7.375 & 0.315 & 0.13 & 12 & $\begin{array}{l}\mathrm{H}, 3-\mathrm{Br}, 4-\mathrm{Br}, 2-\mathrm{Cl}, 3-\mathrm{Cl}, 4-\mathrm{Cl}, 2-\mathrm{OH}, 4-\mathrm{OH}, \\
2-\mathrm{OCH}_{3}, 4-\mathrm{CH}_{3}, 2-\mathrm{NO}_{2}, 3-\mathrm{NO}_{2}\end{array}$ \\
\hline & $\sigma_{\mathrm{I}}$ & 0.906 & 7.916 & 0.573 & 0.17 & 12 & $\begin{array}{l}\mathrm{H}, 3-\mathrm{Br}, 4-\mathrm{Br}, 2-\mathrm{Cl}, 3-\mathrm{Cl}, 4-\mathrm{Cl}, 2-\mathrm{OH}, 4-\mathrm{OH}, \\
2-\mathrm{OCH}_{3}, 4-\mathrm{CH}_{3}, 2-\mathrm{NO}_{2}, 3-\mathrm{NO}_{2}\end{array}$ \\
\hline & $\overline{\sigma_{\mathrm{R}}}$ & 0.984 & 7.543 & 0.703 & 0.11 & 12 & $\begin{array}{l}\mathrm{H}, 3-\mathrm{Br}, 4-\mathrm{Br}, 2-\mathrm{Cl}, 3-\mathrm{Cl}, 4-\mathrm{Cl}, 2-\mathrm{OH}, 4-\mathrm{OH}, \\
2-\mathrm{OCH}_{3}, 4-\mathrm{CH}_{3}, 2-\mathrm{NO}_{2}, 3-\mathrm{NO}_{2}\end{array}$ \\
\hline & $\mathrm{F}$ & 0.955 & 7.179 & 0.615 & 0.17 & 12 & $\begin{array}{l}\mathrm{H}, 3-\mathrm{Br}, 4-\mathrm{Br}, 2-\mathrm{Cl}, 3-\mathrm{Cl}, 4-\mathrm{Cl}, 2-\mathrm{OH}, 4-\mathrm{OH} \text {, } \\
2-\mathrm{OCH}_{3}, 4-\mathrm{CH}_{3}, 2-\mathrm{NO}_{2}, 3-\mathrm{NO}_{2}\end{array}$ \\
\hline & $\mathrm{R}$ & 0.979 & 7.550 & 0.589 & 0.13 & 12 & $\begin{array}{l}\mathrm{H}, 3-\mathrm{Br}, 4-\mathrm{Br}, 2-\mathrm{Cl}, 3-\mathrm{Cl}, 4-\mathrm{Cl}, 2-\mathrm{OH}, 4-\mathrm{OH}, \\
2-\mathrm{OCH}_{3}, 4-\mathrm{CH}_{3}, 2-\mathrm{NO}_{2}, 3-\mathrm{NO}_{2}\end{array}$ \\
\hline \multirow[t]{6}{*}{$\mathrm{H}_{\beta}$} & $\sigma$ & 0.900 & 7.745 & 0.407 & 0.37 & 12 & $\begin{array}{l}\mathrm{H}, 3-\mathrm{Br}, 4-\mathrm{Br}, 2-\mathrm{Cl}, 3-\mathrm{Cl}, 4-\mathrm{Cl}, 2-\mathrm{OH}, 4-\mathrm{OH}, \\
2-\mathrm{OCH}_{3}, 4-\mathrm{CH}_{3}, 2-\mathrm{NO}_{2}, 3-\mathrm{NO}_{2}\end{array}$ \\
\hline & $\sigma^{+}$ & 0.902 & 7.786 & 0.199 & 0.39 & 12 & $\begin{array}{l}\mathrm{H}, 3-\mathrm{Br}, 4-\mathrm{Br}, 2-\mathrm{Cl}, 3-\mathrm{Cl}, 4-\mathrm{Cl}, 2-\mathrm{OH}, 4-\mathrm{OH}, \\
2-\mathrm{OCH}_{3}, 4-\mathrm{CH}_{3}, 2-\mathrm{NO}_{2}, 3-\mathrm{NO}_{2}\end{array}$ \\
\hline & $\sigma_{\mathrm{I}}$ & 0.816 & 7.703 & 0.286 & 0.40 & 12 & $\begin{array}{l}\mathrm{H}, 3-\mathrm{Br}, 4-\mathrm{Br}, 2-\mathrm{Cl}, 3-\mathrm{Cl}, 4-\mathrm{Cl}, 2-\mathrm{OH}, 4-\mathrm{OH}, \\
\text { 2- } \mathrm{OCH}_{3}, 4-\mathrm{CH}_{3}, 2-\mathrm{NO}_{2}, 3-\mathrm{NO}_{2}\end{array}$ \\
\hline & $\sigma_{\mathrm{R}}$ & 0.905 & 7.975 & 0.855 & 0.33 & 12 & $\begin{array}{l}\mathrm{H}, 3-\mathrm{Br}, 4-\mathrm{Br}, 2-\mathrm{Cl}, 3-\mathrm{Cl}, 4-\mathrm{Cl}, 2-\mathrm{OH}, 4-\mathrm{OH}, \\
2-\mathrm{OCH}_{3}, 4-\mathrm{CH}_{3}, 2-\mathrm{NO}_{2}, 3-\mathrm{NO}_{2}\end{array}$ \\
\hline & $\mathrm{F}$ & 0.824 & 7.635 & 0.464 & 0.39 & 12 & $\begin{array}{l}\mathrm{H}, 3-\mathrm{Br}, 4-\mathrm{Br}, 2-\mathrm{Cl}, 3-\mathrm{Cl}, 4-\mathrm{Cl}, 2-\mathrm{OH}, 4-\mathrm{OH}, \\
2-\mathrm{OCH}_{3}, 4-\mathrm{CH}_{3}, 2-\mathrm{NO}_{2}, 3-\mathrm{NO}_{2}\end{array}$ \\
\hline & $\mathrm{R}$ & 0.904 & 7.960 & 0.630 & 0.36 & 12 & $\begin{array}{l}\mathrm{H}, 3-\mathrm{Br}, 4-\mathrm{Br}, 2-\mathrm{Cl}, 3-\mathrm{Cl}, 4-\mathrm{Cl}, 2-\mathrm{OH}, 4-\mathrm{OH}, \\
2-\mathrm{OCH}_{3}, 4-\mathrm{CH}_{3}, 2-\mathrm{NO}_{2}, 3-\mathrm{NO}_{2}\end{array}$ \\
\hline \multirow[t]{6}{*}{$\mathrm{H}_{\beta^{\prime}}$} & $\sigma$ & 0.967 & 8.122 & 0.309 & 0.13 & 12 & $\begin{array}{l}\mathrm{H}, 3-\mathrm{Br}, 4-\mathrm{Br}, 2-\mathrm{Cl}, 3-\mathrm{Cl}, 4-\mathrm{Cl}, 2-\mathrm{OH}, 4-\mathrm{OH}, \\
2-\mathrm{OCH}_{3}, 4-\mathrm{CH}_{3}, 2-\mathrm{NO}_{2}, 3-\mathrm{NO}_{2}\end{array}$ \\
\hline & $\sigma^{+}$ & 0.954 & 8.150 & 0.180 & 0.15 & 12 & $\begin{array}{l}\mathrm{H}, 3-\mathrm{Br}, 4-\mathrm{Br}, 2-\mathrm{Cl}, 3-\mathrm{Cl}, 4-\mathrm{Cl}, 2-\mathrm{OH}, 4-\mathrm{OH}, \\
2-\mathrm{OCH}_{3}, 4-\mathrm{CH}_{3}, 2-\mathrm{NO}_{2}, 3-\mathrm{NO}_{2}\end{array}$ \\
\hline & $\sigma_{\mathrm{I}}$ & 0.833 & 8.770 & 0.275 & 0.17 & 12 & $\begin{array}{l}\mathrm{H}, 3-\mathrm{Br}, 4-\mathrm{Br}, 2-\mathrm{Cl}, 3-\mathrm{Cl}, 4-\mathrm{Cl}, 2-\mathrm{OH}, 4-\mathrm{OH}, \\
2-\mathrm{OCH}_{3}, 4-\mathrm{CH}_{3}, 2-\mathrm{NO}_{2}, 3-\mathrm{NO}_{2}\end{array}$ \\
\hline & $\overline{\sigma_{\mathrm{R}}}$ & 0.977 & 8.278 & 0.558 & 0.11 & 12 & $\begin{array}{l}\mathrm{H}, 3-\mathrm{Br}, 4-\mathrm{Br}, 2-\mathrm{Cl}, 3-\mathrm{Cl}, 4-\mathrm{Cl}, 2-\mathrm{OH}, 4-\mathrm{OH}, \\
2-\mathrm{OCH}_{3}, 4-\mathrm{CH}_{3}, 2-\mathrm{NO}_{2}, 3-\mathrm{NO}_{2}\end{array}$ \\
\hline & $F$ & 0.803 & 8.054 & 0.317 & 0.17 & 12 & $\begin{array}{l}\mathrm{H}, 3-\mathrm{Br}, 4-\mathrm{Br}, 2-\mathrm{Cl}, 3-\mathrm{Cl}, 4-\mathrm{Cl}, 2-\mathrm{OH}, 4-\mathrm{OH}, \\
2-\mathrm{OCH}_{3}, 4-\mathrm{CH}_{3}, 2-\mathrm{NO}_{2}, 3-\mathrm{NO}_{2}\end{array}$ \\
\hline & $\bar{R}$ & 0.907 & 8.281 & 0.464 & 0.12 & 12 & $\begin{array}{l}\mathrm{H}, 3-\mathrm{Br}, 4-\mathrm{Br}, 2-\mathrm{Cl}, 3-\mathrm{Cl}, 4-\mathrm{Cl}, 2-\mathrm{OH}, 4-\mathrm{OH}, \\
2-\mathrm{OCH}_{3}, 4-\mathrm{CH}_{3}, 2-\mathrm{NO}_{2}, 3-\mathrm{NO}_{2}\end{array}$ \\
\hline
\end{tabular}

$\mathrm{r}=$ correlation coefficient; $\mathrm{I}=$ intercept; $\rho=$ slope; $\mathrm{s}=$ number of correlated derivatives parameters.

While seeking they are worthful and produce satisfactory correlation in the multi-regression analysis with $\sigma_{\mathrm{I}}$ and $\sigma_{\mathrm{R}}$ or $\mathrm{F}$ and $\mathrm{R}$ parameters[33]. The generated multi-regression analysis equations are given in (9-14).

$$
\begin{gathered}
\delta \mathrm{H}_{\alpha}(\mathrm{ppm})=7.396( \pm 0.06)+0.350( \pm 0.137) \sigma_{\mathrm{I}}+0.601( \pm 0.120) \sigma_{\mathrm{R}} \\
(R=0.991, \mathrm{n}=12, P>95 \%) \\
\delta \mathrm{H}_{\alpha}(\mathrm{ppm})=7.372( \pm 0.428)+0.428( \pm 0.194) \mathrm{F}+0.506( \pm 0.988) \mathrm{R} \\
(R=0.988, \mathrm{n}=12, P>95 \%) \\
\delta \mathrm{H}_{\beta}(\mathrm{ppm})=7.992( \pm 0.256)-0.038( \pm 0.005) \sigma_{\mathrm{I}}+0.867( \pm 0.245) \sigma_{\mathrm{R}} \\
(R=0.955, \mathrm{n}=12, P>95 \%) \\
\delta \mathrm{H}_{\beta}(\mathrm{ppm})=7.857( \pm 0.283)+0.024( \pm 0.052) \mathrm{F}+0.582( \pm 0.042) \mathrm{R} \\
(R=0.947, \mathrm{n}=12, P>90 \%) \\
\delta \mathrm{H}_{\beta^{\prime}}(\mathrm{ppm})=8.248( \pm 0.088)+0.070( \pm 0.011) \sigma_{\mathrm{I}}+0.535 \quad( \pm 0.157) \sigma_{\mathrm{R}} \\
(R=0.978, \mathrm{n}=12, P>95 \%) \\
\delta \mathrm{H}_{\beta^{\prime}}(\mathrm{ppm})=8.216( \pm 0.095)+0.157( \pm 0.202) \mathrm{F}+0.433( \pm 0.146) \mathrm{R} \\
(R=0.974, \mathrm{n}=12, P>95 \%)
\end{gathered}
$$




\subsection{2. ${ }^{13} \mathrm{C}$ NMR spectral study}

The assigned chemical shifts of $\left.\delta \mathrm{CO}, \mathrm{C}_{\alpha}, \mathrm{C}_{\beta}, \mathrm{CO}_{\text {ester }}, \mathrm{C}_{\alpha^{\prime}}, \mathrm{C}_{\beta^{\prime}} 9 \mathrm{ppm}\right)$ of $(E)$-2-benzyloxy-(3methoxyacrylate)-1-naphthyl enones have been assigned and tabulated in Table 2. These chemical shifts were correlated with Hammett substituent constants, $\mathrm{F}$ and $\mathrm{R}$ parameters using single and multi-regression analysis[2, 6, 14, 19, 24-32]. The results of statistical analyses are presented in Table 5. From the Table 5, the chemical shifts of $\delta \mathrm{CO}, \mathrm{C}_{\alpha}, \mathrm{C}_{\beta}$ of enone moieties are satisfactorily correlated with Hammett substituent constants, F and R parameters. The chemical shifts (ppm) of $\delta \mathrm{CO}_{\text {ester }}$ is correlated satisfactorily with Hammett $\sigma$ and $\sigma^{+}$constants. The correlation of the chemical shifts (ppm) of $\delta C_{\alpha^{\prime}}$ is satisfactory with Hammett substituent constants, $F$ and $R$ parameters. The correlation of the chemical shifts (ppm) of $\delta \mathrm{C}_{\beta^{\prime}}$ is satisfactory with Hammett $\sigma$, $\sigma^{+}, \sigma_{\mathrm{I}}$ constants and $\mathrm{F}$ parameters. The remaining Hammett sigma constants, $\mathrm{F}$ and $\mathrm{R}$ parameters were fail in correlation for the $\delta \mathrm{CO}_{\text {ester }}$ and $\delta \mathrm{C}_{\beta^{\prime}}$ chemical shifts (ppm). All correlations gave positive $\rho$ values. This implies that the normal substituent effect operates in all systems. The failure in the correlation is due to the reasons stated earlier and associated with the resonance-conjugative structure as shown in Fig. 2.

Some of the single parameter correlations were fail for the $\delta C_{\beta}$ and $\delta C_{\beta^{\prime}}$ carbon chemical shifts (ppm) with Hammett substituent constants, F and R parameters. While seeking they are worthful and produce satisfactory correlation in the multi-regression analysis[33] with $\sigma_{I}$ and $\sigma_{R}$ or $\mathrm{F}$ and $\mathrm{R}$ parameters. The generated multi-regression analysis equations for the $\delta \mathrm{CO}_{\text {ester }}, \delta \mathrm{C}_{\beta}$ and $\delta \mathrm{C}_{\beta^{\prime}}$ carbons are given in (15-26).

$$
\begin{gathered}
\delta \mathrm{CO}(\mathrm{ppm})=186.86( \pm 1.228)+7.608\left( \pm 2.471 \sigma_{\mathrm{I}}+3.417( \pm 2.198) \sigma_{\mathrm{R}}\right. \\
(R=0.980, \mathrm{n}=12, P>95 \%) \\
\delta \mathrm{CO}(\mathrm{ppm})=186.59( \pm 1.346)+8.380( \pm 2.766) \mathrm{F}+2.884( \pm 0.200) \mathrm{R} \\
(R=0.978, \mathrm{n}=12, P>95 \%) \\
\delta \mathrm{C}_{\alpha}(\mathrm{ppm})=123.88( \pm 0.368)+1.171( \pm 0.721) \sigma_{\mathrm{I}}+1.388( \pm 0.064) \sigma_{\mathrm{R}} \\
(R=0.979, \mathrm{n}=12, P>95 \%)
\end{gathered}
$$

\begin{tabular}{|c|c|c|c|c|c|c|c|}
\hline Freq. & Constt. & $\mathrm{r}$ & I & $\rho$ & $\mathrm{s}$ & $\mathrm{n}$ & Correlated derivatives \\
\hline \multirow[t]{6}{*}{$\mathrm{CO}$} & $\sigma$ & 0.977 & 188.20 & 5.194 & 1.75 & 12 & $\begin{array}{l}\mathrm{H}, 3-\mathrm{Br}, 4-\mathrm{Br}, 2-\mathrm{Cl}, 3-\mathrm{Cl}, 4-\mathrm{Cl}, 2-\mathrm{OH}, 4-\mathrm{OH}, 2-\mathrm{OCH}_{3} \text {, } \\
\text { 4- } \mathrm{CH}_{3}, 2-\mathrm{NO}_{2}, 3-\mathrm{NO}_{2}\end{array}$ \\
\hline & $\sigma^{+}$ & 0.969 & 188.63 & 3.469 & 1.98 & 12 & $\begin{array}{l}\mathrm{H}, 3-\mathrm{Br}, 4-\mathrm{Br}, 2-\mathrm{Cl}, 3-\mathrm{Cl}, 4-\mathrm{Cl}, 2-\mathrm{OH}, 4-\mathrm{OH}, 2-\mathrm{OCH}_{3} \text {, } \\
\text { 4- } \mathrm{CH}_{3}, 2-\mathrm{NO}_{2}, 3-\mathrm{NO}_{2}\end{array}$ \\
\hline & $\sigma_{\mathrm{I}}$ & 0.974 & 185.72 & 8.873 & 1.83 & 12 & $\begin{array}{l}\mathrm{H}, 3-\mathrm{Br}, 4-\mathrm{Br}, 2-\mathrm{Cl}, 3-\mathrm{Cl}, 4-\mathrm{Cl}, 2-\mathrm{OH}, 4-\mathrm{OH}, 2-\mathrm{OCH}_{3} \text {, } \\
4-\mathrm{CH}_{3}, 2-\mathrm{NO}_{2}, 3-\mathrm{NO}_{2}\end{array}$ \\
\hline & $\sigma_{\mathrm{R}}$ & 0.905 & 190.08 & 5.623 & 2.33 & 12 & $\begin{array}{l}\mathrm{H}, 3-\mathrm{Br}, 4-\mathrm{Br}, 2-\mathrm{Cl}, 3-\mathrm{Cl}, 4-\mathrm{Cl}, 2-\mathrm{OH}, 4-\mathrm{OH}, 2-\mathrm{OCH}_{3} \text {, } \\
\text { 4- } \mathrm{CH}_{3}, 2-\mathrm{NO}_{2}, 3-\mathrm{NO}_{2}\end{array}$ \\
\hline & $\mathrm{F}$ & 0.971 & 185.45 & 9.443 & 1.88 & 12 & $\begin{array}{l}\mathrm{H}, 3-\mathrm{Br}, 4-\mathrm{Br}, 2-\mathrm{Cl}, 3-\mathrm{Cl}, 4-\mathrm{Cl}, 2-\mathrm{OH}, 4-\mathrm{OH}, 2-\mathrm{OCH}_{3} \text {, } \\
\text { 4- } \mathrm{CH}_{3}, 2-\mathrm{NO}_{2}, 3-\mathrm{NO}_{2}\end{array}$ \\
\hline & $\mathrm{R}$ & 0.843 & 190.07 & 4.508 & 2.41 & 12 & $\begin{array}{l}\mathrm{H}, 3-\mathrm{Br}, 4-\mathrm{Br}, 2-\mathrm{Cl}, 3-\mathrm{Cl}, 4-\mathrm{Cl}, 2-\mathrm{OH}, 4-\mathrm{OH}, 2-\mathrm{OCH}_{3} \text {, } \\
\text { 4- } \mathrm{CH}_{3}, 2-\mathrm{NO}_{2}, 3-\mathrm{NO}_{2}\end{array}$ \\
\hline \multirow[t]{6}{*}{$\overline{\mathrm{C}_{\alpha}}$} & $\sigma$ & 0.967 & 124.04 & 1.298 & 0.57 & 12 & $\begin{array}{l}\mathrm{H}, 3-\mathrm{Br}, 4-\mathrm{Br}, 2-\mathrm{Cl}, 3-\mathrm{Cl}, 4-\mathrm{Cl}, 2-\mathrm{OH}, 4-\mathrm{OH}, 2-\mathrm{OCH}_{3} \text {, } \\
\text { 4- } \mathrm{CH}_{3}, 2-\mathrm{NO}_{2}, 3-\mathrm{NO}_{2}\end{array}$ \\
\hline & $\sigma^{+}$ & 0.954 & 124.16 & 0.774 & 0.65 & 12 & $\begin{array}{l}\mathrm{H}, 3-\mathrm{Br}, 4-\mathrm{Br}, 2-\mathrm{Cl}, 3-\mathrm{Cl}, 4-\mathrm{Cl}, 2-\mathrm{OH}, 4-\mathrm{OH}, 2-\mathrm{OCH}_{3} \text {, } \\
\text { 4- } \mathrm{CH}_{3}, 2-\mathrm{NO}_{2}, 3-\mathrm{NO}_{2}\end{array}$ \\
\hline & $\sigma_{\mathrm{I}}$ & 0.960 & 123.42 & 2.223 & 0.58 & 12 & $\begin{array}{l}\mathrm{H}, 3-\mathrm{Br}, 4-\mathrm{Br}, 2-\mathrm{Cl}, 3-\mathrm{Cl}, 4-\mathrm{Cl}, 2-\mathrm{OH}, 4-\mathrm{OH}, 2-\mathrm{OCH}_{3}, \\
4-\mathrm{CH}_{3}, 2-\mathrm{NO}_{2}, 3-\mathrm{NO}_{2}\end{array}$ \\
\hline & $\sigma_{\mathrm{R}}$ & 0.963 & 124.61 & 1.880 & 0.60 & 12 & $\begin{array}{l}\mathrm{H}, 3-\mathrm{Br}, 4-\mathrm{Br}, 2-\mathrm{Cl}, 3-\mathrm{Cl}, 4-\mathrm{Cl}, 2-\mathrm{OH}, 4-\mathrm{OH}, 2-\mathrm{OCH}_{3} \text {, } \\
\text { 4- } \mathrm{CH}_{3}, 2-\mathrm{NO}_{2}, 3-\mathrm{NO}_{2}\end{array}$ \\
\hline & $F$ & 0.960 & 123.37 & 2.340 & 0.60 & 12 & $\begin{array}{l}\mathrm{H}, 3-\mathrm{Br}, 4-\mathrm{Br}, 2-\mathrm{Cl}, 3-\mathrm{Cl}, 4-\mathrm{Cl}, 2-\mathrm{OH}, 4-\mathrm{OH}, 2-\mathrm{OCH}_{3} \text {, } \\
\text { 4- } \mathrm{CH}_{3}, 2-\mathrm{NO}_{2}, 3-\mathrm{NO}_{2}\end{array}$ \\
\hline & $\mathrm{R}$ & 0.961 & 124.63 & 1.632 & 0.61 & 12 & $\begin{array}{l}\mathrm{H}, 3-\mathrm{Br}, 4-\mathrm{Br}, 2-\mathrm{Cl}, 3-\mathrm{Cl}, 4-\mathrm{Cl}, 2-\mathrm{OH}, 4-\mathrm{OH}, 2-\mathrm{OCH}_{3}, \\
4-\mathrm{CH}_{3}, 2-\mathrm{NO}_{2}, 3-\mathrm{NO}_{2}\end{array}$ \\
\hline
\end{tabular}

Table 5. Results of statistical analysis of ${ }^{13} \mathrm{C}$ NMR chemical shifts $(\delta, \mathrm{ppm})$ of $\mathrm{CO}, \mathrm{C}_{\alpha}, \mathrm{C}_{\beta}$ of enone moiety, $\mathrm{CO}, \mathrm{C}_{\alpha^{\prime}}, \mathrm{C}_{\beta^{\prime}}$ ester moiety of 1-(2-benzyloxy-(3-methoxyacrylate))-1-naphthyl enones with Hammett $\sigma, \sigma^{+}, \sigma_{\mathrm{I}}, \sigma_{\mathrm{R}}$, constants, $\mathrm{F}$ and $\mathrm{R}$ parameters. 


\begin{tabular}{|c|c|c|c|c|c|c|c|}
\hline \multirow[t]{6}{*}{$\mathrm{C}_{\beta}$} & $\sigma$ & 0.972 & 139.52 & 2.508 & 0.97 & 12 & $\begin{array}{l}\mathrm{H}, 3-\mathrm{Br}, 4-\mathrm{Br}, 2-\mathrm{Cl}, 3-\mathrm{Cl}, 4-\mathrm{Cl}, 2-\mathrm{OH}, 4-\mathrm{OH}, 2-\mathrm{OCH}_{3} \text {, } \\
4-\mathrm{CH}_{3}, 2-\mathrm{NO}_{2}, 3-\mathrm{NO}_{2}\end{array}$ \\
\hline & $\sigma^{+}$ & 0.970 & 139.72 & 1.810 & 1.00 & 12 & $\begin{array}{l}\mathrm{H}, 3-\mathrm{Br}, 4-\mathrm{Br}, 2-\mathrm{Cl}, 3-\mathrm{Cl}, 4-\mathrm{Cl}, 2-\mathrm{OH}, 4-\mathrm{OH}, 2-\mathrm{OCH}_{3} \text {, } \\
\text { 4- } \mathrm{CH}_{3}, 2-\mathrm{NO}_{2}, 3-\mathrm{NO}_{2}\end{array}$ \\
\hline & $\sigma_{\mathrm{I}}$ & 0.905 & 138.63 & 3.453 & 1.17 & 12 & $\begin{array}{l}\mathrm{H}, 3-\mathrm{Br}, 4-\mathrm{Br}, 2-\mathrm{Cl}, 3-\mathrm{Cl}, 4-\mathrm{Cl}, 2-\mathrm{OH}, 4-\mathrm{OH}, 2-\mathrm{OCH}_{3} \text {, } \\
4-\mathrm{CH}_{3}, 2-\mathrm{NO}_{2}, 3-\mathrm{NO}_{2}\end{array}$ \\
\hline & $\sigma_{\mathrm{R}}$ & 0.978 & 140.75 & 4.271 & 0.87 & 12 & $\begin{array}{l}\mathrm{H}, 3-\mathrm{Br}, 4-\mathrm{Br}, 2-\mathrm{Cl}, 3-\mathrm{Cl}, 4-\mathrm{Cl}, 2-\mathrm{OH}, 4-\mathrm{OH}, 2-\mathrm{OCH}_{3} \text {, } \\
4-\mathrm{CH}_{3}, 2-\mathrm{NO}_{2}, 3-\mathrm{NO}_{2}\end{array}$ \\
\hline & $\mathrm{F}$ & 0.915 & 138.68 & 3.476 & 1.21 & 12 & $\begin{array}{l}\mathrm{H}, 3-\mathrm{Br}, 4-\mathrm{Br}, 2-\mathrm{Cl}, 3-\mathrm{Cl}, 4-\mathrm{Cl}, 2-\mathrm{OH}, 4-\mathrm{OH}, 2-\mathrm{OCH}_{3} \text {, } \\
4-\mathrm{CH}_{3}, 2-\mathrm{NO}_{2}, 3-\mathrm{NO}_{2}\end{array}$ \\
\hline & $\mathrm{R}$ & 0.971 & 140.78 & 3.616 & 0.93 & 12 & $\begin{array}{l}\mathrm{H}, 3-\mathrm{Br}, 4-\mathrm{Br}, 2-\mathrm{Cl}, 3-\mathrm{Cl}, 4-\mathrm{Cl}, 2-\mathrm{OH}, 4-\mathrm{OH}, 2-\mathrm{OCH}_{3} \text {, } \\
4-\mathrm{CH}_{3}, 2-\mathrm{NO}_{2}, 3-\mathrm{NO}_{2}\end{array}$ \\
\hline \multirow[t]{6}{*}{$\mathrm{CO}_{\text {ester }}$} & $\sigma$ & 0.903 & 164.56 & 0.856 & 1.25 & 12 & $\begin{array}{l}\mathrm{H}, 3-\mathrm{Br}, 4-\mathrm{Br}, 2-\mathrm{Cl}, 3-\mathrm{Cl}, 4-\mathrm{Cl}, 2-\mathrm{OH}, 4-\mathrm{OH}, 2-\mathrm{OCH}_{3} \text {, } \\
4-\mathrm{CH}_{3}, 2-\mathrm{NO}_{2}, 3-\mathrm{NO}_{2}\end{array}$ \\
\hline & $\sigma^{+}$ & 0.900 & 164.65 & 0.365 & 1.28 & 12 & $\begin{array}{l}\mathrm{H}, 3-\mathrm{Br}, 4-\mathrm{Br}, 2-\mathrm{Cl}, 3-\mathrm{Cl}, 4-\mathrm{Cl}, 2-\mathrm{OH}, 4-\mathrm{OH}, 2-\mathrm{OCH}_{3} \text {, } \\
4-\mathrm{CH}_{3}, 2-\mathrm{NO}_{2}, 3-\mathrm{NO}_{2}\end{array}$ \\
\hline & $\sigma_{\mathrm{I}}$ & 0.812 & 164.24 & 1.222 & 2.26 & 12 & $\begin{array}{l}\mathrm{H}, 3-\mathrm{Br}, 4-\mathrm{Br}, 2-\mathrm{Cl}, 3-\mathrm{Cl}, 4-\mathrm{Cl}, 2-\mathrm{OH}, 4-\mathrm{OH}, 2-\mathrm{OCH}_{3} \text {, } \\
4-\mathrm{CH}_{3}, 2-\mathrm{NO}_{2}, 3-\mathrm{NO}_{2}\end{array}$ \\
\hline & $\sigma_{\mathrm{R}}$ & 0.705 & 164.74 & 0.269 & 2.29 & 12 & $\begin{array}{l}\mathrm{H}, 3-\mathrm{Br}, 4-\mathrm{Br}, 2-\mathrm{Cl}, 3-\mathrm{Cl}, 4-\mathrm{Cl}, 2-\mathrm{OH}, 4-\mathrm{OH}, 2-\mathrm{OCH}_{3} \text {, } \\
\text { 4- } \mathrm{CH}_{3}, 2-\mathrm{NO}_{2}, 3-\mathrm{NO}_{2}\end{array}$ \\
\hline & $\mathrm{F}$ & 0.729 & 164.03 & 0.800 & 1.20 & 12 & $\begin{array}{l}\mathrm{H}, 3-\mathrm{Br}, 4-\mathrm{Br}, 2-\mathrm{Cl}, 3-\mathrm{Cl}, 4-\mathrm{Cl}, 2-\mathrm{OH}, 4-\mathrm{OH}, 2-\mathrm{OCH}_{3} \text {, } \\
4-\mathrm{CH}_{3}, 2-\mathrm{NO}_{2}, 3-\mathrm{NO}_{2}\end{array}$ \\
\hline & $\mathrm{R}$ & 0.803 & 164.76 & 0.280 & 1.29 & 12 & $\begin{array}{l}\mathrm{H}, 3-\mathrm{Br}, 4-\mathrm{Br}, 2-\mathrm{Cl}, 3-\mathrm{Cl}, 4-\mathrm{Cl}, 2-\mathrm{OH}, 4-\mathrm{OH}, 2-\mathrm{OCH}_{3} \text {, } \\
4-\mathrm{CH}_{3}, 2-\mathrm{NO}_{2}, 3-\mathrm{NO}_{2}\end{array}$ \\
\hline \multirow[t]{6}{*}{$\mathrm{C}_{\alpha^{\prime}}$} & $\sigma$ & 0.989 & 112.07 & 9.499 & 1.95 & 12 & $\begin{array}{l}\mathrm{H}, 3-\mathrm{Br}, 4-\mathrm{Br}, 2-\mathrm{Cl}, 3-\mathrm{Cl}, 4-\mathrm{Cl}, 2-\mathrm{OH}, 4-\mathrm{OH}, 2-\mathrm{OCH}_{3} \text {, } \\
\text { 4- } \mathrm{CH}_{3}, 2-\mathrm{NO}_{2}, 3-\mathrm{NO}_{2}\end{array}$ \\
\hline & $\sigma^{+}$ & 0.900 & 11.78 & 7.088 & 1.89 & 12 & $\begin{array}{l}\mathrm{H}, 3-\mathrm{Br}, 4-\mathrm{Br}, 2-\mathrm{Cl}, 3-\mathrm{Cl}, 4-\mathrm{Cl}, 2-\mathrm{OH}, 4-\mathrm{OH}, 2-\mathrm{OCH}_{3} \text {, } \\
4-\mathrm{CH}_{3}, 2-\mathrm{NO}_{2}, 3-\mathrm{NO}_{2}\end{array}$ \\
\hline & $\sigma_{\mathrm{I}}$ & 0.903 & 108.85 & 12.631 & 3.21 & 12 & $\begin{array}{l}\mathrm{H}, 3-\mathrm{Br}, 4-\mathrm{Br}, 2-\mathrm{Cl}, 3-\mathrm{Cl}, 4-\mathrm{Cl}, 2-\mathrm{OH}, 4-\mathrm{OH}, 2-\mathrm{OCH}_{3} \text {, } \\
4-\mathrm{CH}_{3}, 2-\mathrm{NO}_{2}, 3-\mathrm{NO}_{2}\end{array}$ \\
\hline & $\sigma_{\mathrm{R}}$ & 0.907 & 116.00 & 12.721 & 2.79 & 12 & $\begin{array}{l}\mathrm{H}, 3-\mathrm{Br}, 4-\mathrm{Br}, 2-\mathrm{Cl}, 3-\mathrm{Cl}, 4-\mathrm{Cl}, 2-\mathrm{OH}, 4-\mathrm{OH}, 2-\mathrm{OCH}_{3} \text {, } \\
4-\mathrm{CH}_{3}, 2-\mathrm{NO}_{2}, 3-\mathrm{NO}_{2}\end{array}$ \\
\hline & $\mathrm{F}$ & 0.961 & 108.31 & 12.610 & 2.07 & 12 & $\begin{array}{l}\mathrm{H}, 3-\mathrm{Br}, 4-\mathrm{Br}, 2-\mathrm{Cl}, 3-\mathrm{Cl}, 4-\mathrm{Cl}, 2-\mathrm{OH}, 4-\mathrm{OH}, 2-\mathrm{OCH}_{3} \text {, } \\
4-\mathrm{CH}_{3}, 2-\mathrm{NO}_{2}, 3-\mathrm{NO}_{2}\end{array}$ \\
\hline & $\mathrm{R}$ & 0.907 & 116.26 & 11.381 & 2.78 & 12 & $\begin{array}{l}\mathrm{H}, 3-\mathrm{Br}, 4-\mathrm{Br}, 2-\mathrm{Cl}, 3-\mathrm{Cl}, 4-\mathrm{Cl}, 2-\mathrm{OH}, 4-\mathrm{OH}, 2-\mathrm{OCH}_{3} \text {, } \\
\text { 4- } \mathrm{CH}_{3}, 2-\mathrm{NO}_{2}, 3-\mathrm{NO}_{2}\end{array}$ \\
\hline \multirow[t]{6}{*}{$\mathrm{C}_{\beta^{\prime}}$} & $\sigma$ & 0.961 & 150.30 & 2.873 & 1.27 & 12 & $\begin{array}{l}\mathrm{H}, 3-\mathrm{Br}, 4-\mathrm{Br}, 2-\mathrm{Cl}, 3-\mathrm{Cl}, 4-\mathrm{Cl}, 2-\mathrm{OH}, 4-\mathrm{OH}, 2-\mathrm{OCH}_{3} \text {, } \\
4-\mathrm{CH}_{3}, 2-\mathrm{NO}_{2}, 3-\mathrm{NO}_{2}\end{array}$ \\
\hline & $\sigma^{+}$ & 0.965 & 150.53 & 2.063 & 1.30 & 12 & $\begin{array}{l}\mathrm{H}, 3-\mathrm{Br}, 4-\mathrm{Br}, 2-\mathrm{Cl}, 3-\mathrm{Cl}, 4-\mathrm{Cl}, 2-\mathrm{OH}, 4-\mathrm{OH}, 2-\mathrm{OCH}_{3} \text {, } \\
4-\mathrm{CH}_{3}, 2-\mathrm{NO}_{2}, 3-\mathrm{NO}_{2}\end{array}$ \\
\hline & $\sigma_{\mathrm{I}}$ & 0.617 & 148.81 & 5.741 & 1.23 & 12 & $\begin{array}{l}\mathrm{H}, 3-\mathrm{Br}, 4-\mathrm{Br}, 2-\mathrm{Cl}, 3-\mathrm{Cl}, 4-\mathrm{Cl}, 2-\mathrm{OH}, 4-\mathrm{OH}, 2-\mathrm{OCH}_{3} \text {, } \\
\text { 4- } \mathrm{CH}_{3}, 2-\mathrm{NO}_{2}, 3-\mathrm{NO}_{2}\end{array}$ \\
\hline & $\sigma_{\mathrm{R}}$ & 0.839 & 151.24 & 2.586 & 1.59 & 12 & $\begin{array}{l}\mathrm{H}, 3-\mathrm{Br}, 4-\mathrm{Br}, 2-\mathrm{Cl}, 3-\mathrm{Cl}, 4-\mathrm{Cl}, 2-\mathrm{OH}, 4-\mathrm{OH}, 2-\mathrm{OCH}_{3} \text {, } \\
4-\mathrm{CH}_{3}, 2-\mathrm{NO}_{2}, 3-\mathrm{NO}_{2}\end{array}$ \\
\hline & $\mathrm{F}$ & 0.964 & 148.71 & 5.236 & 1.32 & 12 & $\begin{array}{l}\mathrm{H}, 3-\mathrm{Br}, 4-\mathrm{Br}, 2-\mathrm{Cl}, 3-\mathrm{Cl}, 4-\mathrm{Cl}, 2-\mathrm{OH}, 4-\mathrm{OH}, 2-\mathrm{OCH}_{3} \text {, } \\
4-\mathrm{CH}_{3}, 2-\mathrm{NO}_{2}, 3-\mathrm{NO}_{2}\end{array}$ \\
\hline & $\mathrm{R}$ & 0.843 & 151.34 & 2.364 & 1.59 & 12 & $\begin{array}{l}\mathrm{H}, 3-\mathrm{Br}, 4-\mathrm{Br}, 2-\mathrm{Cl}, 3-\mathrm{Cl}, 4-\mathrm{Cl}, 2-\mathrm{OH}, 4-\mathrm{OH}, 2-\mathrm{OCH}_{3} \text {, } \\
4-\mathrm{CH}_{3}, 2-\mathrm{NO}_{2}, 3-\mathrm{NO}_{2}\end{array}$ \\
\hline
\end{tabular}

$\mathrm{r}=$ correlation coefficient; $\mathrm{I}=$ intercept $\rho=$ slope; $\mathrm{s}=$ number of correlated derivatives

$$
\begin{gathered}
\delta \mathrm{C}_{\alpha}(\mathrm{ppm})=123.85( \pm 0.381)+1.876( \pm 0.731) \mathrm{F}+1.278( \pm 0.563) \mathrm{R} \\
(R=0.978, \mathrm{n}=12, P>95 \%) \\
\delta \mathrm{C}_{\beta}(\mathrm{ppm})=139.85( \pm 0.559)+2.099( \pm 1.124) \sigma_{\mathrm{I}}+3.660( \pm 0.992) \sigma_{\mathrm{R}} \\
(R=0.985, \mathrm{n}=12, P>95 \%) \\
\delta \mathrm{C}_{\beta}(\mathrm{ppm})=139.82( \pm 0.640)+2.305( \pm 1.317) \mathrm{F}+3.169( \pm 0.952) \mathrm{R} \\
(R=0.982, \mathrm{n}=12, P>95 \%) \\
\delta \mathrm{CO}_{\text {ester }}(\mathrm{ppm})=164.21( \pm 0.957)+1.206( \pm 0.192) \sigma_{\mathrm{I}}+0.097( \pm 0.001) \sigma_{\mathrm{R}} \\
(R=0.921, \mathrm{n}=12, P>90 \%)
\end{gathered}
$$




$$
\begin{gathered}
\delta \mathrm{CO}_{\text {ester }}(\mathrm{ppm})=164.00( \pm 0.981)+1.827( \pm 0.021) \mathrm{F}+0.073( \pm 0.0 .001) \mathrm{R} \\
(R=0.988, \mathrm{n}=12, P>95 \%) \\
\delta \mathrm{C}_{\alpha^{\prime}}(\mathrm{ppm})=112.240( \pm 1.150)+8.876( \pm 3.058) \sigma_{\mathrm{I}}+110.149( \pm 2.705) \sigma_{\mathrm{R}} \\
(R=0.988, \mathrm{n}=12, P>95 \%) \\
\delta \mathrm{C}_{\alpha^{\prime}}(\mathrm{ppm})=112.507( \pm 1.640)+9.508( \pm 3.371) \mathrm{F}+9.628( \pm 2.438) \mathrm{R} \\
(R=0.987, \mathrm{n}=12, P>95 \%) \\
\delta \mathrm{C}_{\beta^{\prime}}(\mathrm{ppm})=149.20( \pm 0.906)+4.807( \pm 1.823) \sigma_{\mathrm{I}}+1.190( \pm 0.161) \sigma_{\mathrm{R}} \\
(R=0.972, \mathrm{n}=12, P>95 \%) \\
\delta \mathrm{C}_{\beta^{\prime}}(\mathrm{ppm})=149.33( \pm 0.994)+4.751( \pm 2.042) \mathrm{F}+1.44( \pm 0.147) \mathrm{R} \\
(R=0.968, \mathrm{n}=12, P>95 \%)
\end{gathered}
$$

\subsection{Antimicrobial activities}

\subsubsection{Antibacterial sensitivity assay}

Antibacterial activity assay of prepared 2-benzyloxy-(3-methoxyacrylate)-1-naphthyl enones were performed using Kirby-Bauer[34] disc diffusion technique. In every petri plate, about $0.5 \mathrm{~mL}$ of the test bacterial sample was spread over uniformly on the solidified Mueller Hinton agar using sterile glass spreader. Then the discs were made with $5 \mathrm{~mm}$ of Whatmann No.1 filter paper, impregnated with the solution of the compounds placed on the medium using sterile forceps. Incubated the plates for $24 \mathrm{~h}$ at $37^{\circ} \mathrm{C}$ by keeping the plates upside down to prevent the collection of water droplets over the medium. After $24 \mathrm{~h}$, the plates were visually examined and the diameter values of the zone of inhibition were measured. Triplicate results were recorded by repeating the same procedure. The measured antibacterial activities of all oxazine are presented in Table 6.

The antibacterial activity assay of all prepared enones were studied against two gram positive pathogenic strains Staphylococcus aureus, Enterococcus faecalis and four gram negative strains Escherichia coli, Klebsiella species, Psuedomonas and Proteus vulgaris. The disc diffusion technique was followed using the Kirby-Bauer[ ]method, at a concentration of $250 \mu \mathrm{g} / \mathrm{mL}$ with Ampicillin and Streptomycin were used as standard drugs. Compounds 4, 6 and 9 showed maximum zone of inhibition against $B$. substilis, with greater than $20 \mathrm{~mm}$ compared to the ketones 1-3, 5 and $\mathbf{1 0}$ are moderately active in 13-19 $\mathrm{mm}$ of zone of inhibition. Enone 9 was active with in 8-12 mm of zone of inhibition and the compounds 7 and 8 are inactive. The enones $\mathbf{1}$ and $\mathbf{6}$ were found to be effective against $S$. aureus within 20-24 mm of zone of inhibition.

Table 6. Antibacterial activities of 1-(2-benzyloxy-(3-methoxyacrylate)-1-naphthyl enones.

\begin{tabular}{|l|l|l|l|l|l|l|l|}
\hline Entry & $\mathbf{X}$ & B.substilis & M.luteus & S.aures & E-coli & P.aeruginosa & K.pneumoniae \\
\hline $\mathbf{1}$ & $\mathrm{H}$ & + & + & ++ & + & ++ & + \\
\hline $\mathbf{2}$ & $3-\mathrm{Br}$ & + & -- & + & --- & + & --- \\
\hline $\mathbf{3}$ & $4-\mathrm{Br}$ & + & + & --- & + & + & + \\
\hline $\mathbf{4}$ & $2-\mathrm{Cl}$ & ++ & + & + & + & --- & + \\
\hline $\mathbf{5}$ & $3-\mathrm{Cl}$ & + & --- & + & ++ & ++ & ++ \\
\hline $\mathbf{6}$ & $4-\mathrm{Cl}$ & ++ & \pm & ++ & \pm & + & + \\
\hline $\mathbf{7}$ & $2-\mathrm{OH}$ & --- & + & + & + & + & + \\
\hline $\mathbf{8}$ & $4-\mathrm{OH}$ & --- & ++ & + & \pm & + & \pm \\
\hline $\mathbf{9}$ & $2-\mathrm{OCH}_{3}$ & \pm & + & + & --- & \pm & \pm \\
\hline $\mathbf{1 0}$ & $4-\mathrm{CH}_{3}$ & + & + & \pm & -- & ++ & \pm \\
\hline $\mathbf{1 1}$ & $2-\mathrm{NO}_{2}$ & --- & ++ & + & ++ & --- & ++ \\
\hline $\mathbf{1 2}$ & $4-\mathrm{NO}_{2}$ & ++ & \pm & --- & --- & + & + \\
\hline
\end{tabular}

Compounds 1-4, 7, 9 and 10 were moderately active within 13-19 $\mathrm{mm}$ of zone of inhibition. The enones $\mathbf{8}$ and $\mathbf{1 2}$ were active within $8-12 \mathrm{~mm}$ of zone of inhibition. The chalcone derivatives $\mathbf{1 , 5}$ and $\mathbf{1 0}$ were more active against Pseudomonas a showing greater than $20 \mathrm{~mm}$ zone of inhibition and the other derivatives 2, 3, 6, 7 and 12 showed the zone of inhibitions between 13-19 $\mathrm{mm}$. Compound 9 was moderately active within $8-12 \mathrm{~mm}$ of zone of inhibition. The ketones $\mathbf{5}$ and $\mathbf{1 1}$ are 
effective against Klebsiella with 20-24 mm zone of inhibition while the other compounds 1-4, 6, 7, 12 and 8-10 were showed a moderate activity within 13-19 and 8-12 mm of zone of inhibitions. The compounds $\mathbf{8}$ and $\mathbf{1 1}$ were active when it is screened against M. luteus at 20-24mm of zone of inhibition. Enones 1, 3, 4, 7, 8 and 10 were active at 13-19 $\mathrm{mm}$ of zone of inhibition. The ketones 8 and 12 have shown $8-12 \mathrm{~mm}$ zone of inhibition. The enones $\mathbf{5}$ and $\mathbf{1 1}$ are active against he and Escherichia coli at 20-24mm of zone of inhibition. The other compounds 1-4, 7, 6 and 8 are moderately effective within 13-19 and 8-12 mm of zone of inhibitions.

\subsubsection{Antifungal sensitivity assay}

Antifungal sensitivity assay of synthesized enones were performed using Kirby-Bauer ${ }^{[34]}$ disc diffusion technique. The PDA medium was prepared and sterilized as above. This is poured (ear bearing heating condition) to the Petri-plate which was already filled with $1 \mathrm{~mL}$ of the fungal species. The plate was rotated clockwise and counter clock-wise for uniform spreading of the species. The discs were impregnated with the test solution. The test solution was prepared by dissolving $15 \mathrm{mg}$ of the oxazine in $1 \mathrm{~mL}$ of DMSO solvent. The medium was allowed to solidify and kept for $24 \mathrm{~h}$. Then the plates were visually examined and the diameter values of zone of inhibition were measured. Triplicate results were recorded by repeating the same procedure. The observed antifungal activities of all oxazine amines are presented in Table 7.

The study of antifungal activities of all 2-benzyloxy-(3-methoxyacrylate)-1-naphthyl enones has been done with Aspergillus niger, M.spp and T. virdi fungal stains and the dilution method was adopted. The drug dilution was kept as $50 \mu \mathrm{g} / \mathrm{mL}$. Griseofulvin has been taken as the standard drug.

Table 7. Antifungal activities 1-(2-benzyloxy-(3-methoxy acrylate)-1-naphthyl enones

\begin{tabular}{|l|l|l|l|l|}
\hline Entry & X & A.niger & M.spp & T. virdi \\
\hline 1 & $\mathrm{H}$ & \pm & + & --- \\
\hline 2 & $3-\mathrm{Br}$ & --- & \pm & + \\
\hline 3 & $4-\mathrm{Br}$ & \pm & + & + \\
\hline 4 & $2-\mathrm{Cl}$ & + & \pm & + \\
\hline 5 & $3-\mathrm{Cl}$ & --- & + & ++ \\
\hline 6 & $4-\mathrm{Cl}$ & + & ++ & + \\
\hline 7 & $2-\mathrm{OH}$ & + & --- & --- \\
\hline 8 & $4-\mathrm{OH}$ & ++ & --- & ++ \\
\hline 9 & $4-\mathrm{OCH}_{3}$ & + & + & --- \\
\hline 10 & $4-\mathrm{CH}_{3}$ & --- & ++ & + \\
\hline 11 & $2-\mathrm{NO}_{2}$ & + & \pm & ++ \\
\hline 12 & $4-\mathrm{NO}_{2}$ & ++ & ++ & + \\
\hline
\end{tabular}

Standard: Griseofulvin and Gentamycin; Duration: $72 \mathrm{~h}$; Control: Methanol; Medium: Potato dextrose agar; ++: No fungal colony; +: One fungal colony; \pm : Two-three fungal colonies; ---: Heavy fungal colony.

The study of antifungal activities of all 2-benzyloxy-(3-methoxyacrylate)-1-naphthyl enones against $A$. niger showed that the two compounds $\mathbf{8}$ and $\mathbf{1 2}$ are effective with $20 \mathrm{~mm}$ as the zone of inhibition in $250 \mu \mathrm{g} / \mathrm{L}$ disc while ketones 4, 6, 7, 9 and 11 are active with 13-19 $\mathrm{mm}$ zone of inhibition and the compound $\mathbf{1}$ and $\mathbf{3}$ was the least active with $8-12 \mathrm{~mm}$ zone of inhibitions. Compounds 6, 10 and 12 are visible against M. spp. species within $20 \mathrm{~mm}$ zone of inhibitions and the development of the fungal colony (2-3 colonies) are recorded for the compounds 2, 4 and 11. The inhibition of compounds 5, 8 and 11 against $T$. virdi were highly active and the enones 2-4, 6, 10 and 11 are active within $8-12 \mathrm{~mm}$ of zone of inhibition. Presence of halogens, methoxy, methyl and nitro substituents are responsible for antimicrobial activities of synthesized enones. 


\subsection{Antioxidant activity}

The antioxidant activities of all synthesized enones have been evaluated by the DPPH radical scavenging effect[35]. The $0.1 \mathrm{M}$ acetate will be prepared by dissolving $1.64 \mathrm{~g}$ of sodium acetate in $15 \mathrm{~mL}$ of water and $150 \mu \mathrm{L}$ of acetic acid. The final volume will be adjusted to $20 \mathrm{~mL}$ by adding water. The $0.2 \mathrm{mmol}$ of DPPH solution will be prepared by dissolving $3.9 \mathrm{~g}$ of DPPH in 50 $\mathrm{mL}$ of ethanol. $\alpha$-Tocofereol ( $1 \mathrm{mg}$ in $10 \mathrm{~mL}$ of ethanol) solution was prepared. A series of test tubes will be arranged with $1.0 \mathrm{~mL}$ of buffer solution mixed with $0.5 \mathrm{~mL}$ of DPPH solution. A series of various concentrations of synthesized enones and $\alpha$-Tocofereol $(1 \mu \mathrm{g}$ in $1 \mathrm{ml}$ of ethanol) will be added to each tube and mixed well. After 30 minutes in room temperature the absorbance of each solution will be measured by UV spectrophotometer at $517 \mathrm{~nm}$. A mixture of buffer solution and ethanol used as the reference for the spectrophotometer. A graph was plotted with the weight of the compound Vs absorptions and IC50 values will be determined. The antioxidant activity will be expressed in terms of $\mathrm{IC}_{50}(\mu \mathrm{g} / \mathrm{mL}$, concentration required to inhibit $\mathrm{DPPH}$ radical formation by $50 \%$ ). $\alpha$-Tocofereol will be used as a positive control. The radical scavenging activity was calculated as,

$\begin{aligned} & \text { DPPH radical scavenging } \\ & \text { activity }(\% \text { of inhibition })\end{aligned}=\frac{\text { Control absorbance }- \text { Sample absorbance }}{\text { Control absorbance }} \times 100$

From the experimental statistical results, the hydroxyl and methoxy substituted enones (7-9) were shown significant antioxidant activity.

\subsection{Insect antifeedant activity}

Generally organic compounds which are having carbonyl, unsaturation and halogen substitutions, they possess insect antifeedant activity. Therefore, the author wishes to examine the insect antifeedant activity of these enone derivatives and found to be active as insect antifeedant. This test was performed with a $4^{\text {th }}$ instar larva Achoea janata $\mathrm{L}$ against castor semilooper, were reared as described on the leaves of castor, Ricinus communis in the laboratory at the temperature range of $26^{\circ} \mathrm{C} \pm 1^{\circ} \mathrm{C}$ and a relative humidity of $75-85 \%$. The leaf - disc bioassay method was used against the $4^{\text {th }}$ instar larvae to measure the antifeedant activity. The $4^{\text {th }}$ instar larvae were selected for testing because the larvae at this stage feed very voraciously.

\subsubsection{Measurement of insect antifeedant activity of 2-benzyloxy-(3-methoxyacrylate)-1- naphthyl enones}

Castor leaf discs of a diameter of $1.85 \mathrm{~cm}$ were punched and intact with the petioles. The synthesized 2-benzyloxy-(3-methoxyacrylate)-1-naphthyl enones were dissolved in acetone at a concentration of $200 \mathrm{ppm}$ dipped for 5 minutes. The leaf discs were air-dried and placed in one litre beaker containing little water in order to facilitate translocation of water. Therefore, the leaf discs remain fresh throughout the duration of the rest, $4^{\text {th }}$ instar larvae of the test insect, which had been preserved on the leaf discs of all 2-benzyloxy-(3-methoxyacrylate)-1-naphthyl enones and allowed to feed on them for $24 \mathrm{~h}$. The area of the leaf disc consumed were measured by Dethler's method[36]. The observed antifeedant activity of 2-benzyloxy-(3-methoxyacrylate)-1-naphthyl enones was presented in Table 8.

The results of the antifeedant activity of 2-benzyloxy-(3-methoxyacrylate)-1-naphthyl enones are presented in Table 8 reveals that compound 6 1-(2-benzyloxy-(3-methoxyacrylate)-1naphthyl)-4-chloro phenyl chalcone was found to reflect satisfactory antifeedant. This test is performed with the insects which are only two-leaf disc soaked under the solution of this compound. Compound $\mathbf{5}$ showed enough antifeedant activity but lesser than $\mathbf{6}$. Further compound 6 was subjected to measure the antifeedant activity at different 50,100,150 ppm concentrations and the observation reveals that as the concentrations decreased, the activity also decreased. It is observed from the results in Table 9 and that the 6 1-(2-benzyloxy-(3-methoxyacrylo)-1- naphthyl)4-chlorophenyl chalcone showed an appreciable antifeedant activity at $150 \mathrm{ppm}$ concentration. 
Table 8. Insect antifeedant activities of 1-(2-benzyloxy-(3-methoxyacrylo)-1-naphthyl)-3substitutedphenyl chalcone

\begin{tabular}{|l|l|l|l|l|l|l|l|l|l|l|l|}
\hline Entry & $\mathrm{X}$ & $\begin{array}{l}4-6 \\
\mathrm{pm}\end{array}$ & $\begin{array}{l}6-8 \\
\mathrm{pm}\end{array}$ & $\begin{array}{l}8-10 \\
\mathrm{pm}\end{array}$ & $\begin{array}{l}10- \\
12 \\
\mathrm{pm}\end{array}$ & $\begin{array}{l}12- \\
6 \\
\mathrm{am}\end{array}$ & $\begin{array}{l}6-8 \\
\mathrm{am}\end{array}$ & $\begin{array}{l}8 \mathrm{am}- \\
12 \mathrm{Nn}\end{array}$ & $\begin{array}{l}12 \mathrm{Nn}- \\
2 \mathrm{pm}\end{array}$ & $\begin{array}{l}2-4 \\
\text { pm }\end{array}$ & $\begin{array}{l}\text { Total leaf } \\
\text { disc } \\
\text { consumed in } \\
24 \mathrm{~h}\end{array}$ \\
\hline $\mathbf{1}$ & $\mathrm{H}$ & 1 & 1 & 0.5 & 0.5 & 0.5 & 1 & 1 & 1 & 1 & 8 \\
\hline $\mathbf{2}$ & $3-\mathrm{Br}$ & 1 & 2 & 0.25 & 1 & 0.1 & 0 & 1 & 1 & 0.5 & 5 \\
\hline $\mathbf{3}$ & $4-\mathrm{Br}$ & 0.5 & 0.25 & 0.25 & 0.5 & 0.5 & 0.5 & 1 & 1 & 0.5 & 5 \\
\hline $\mathbf{4}$ & $2-\mathrm{Cl}$ & 0.5 & 0.5 & 0.25 & 1 & 0.5 & 0.5 & 0.25 & 0.25 & 0.25 & 4 \\
\hline $\mathbf{5}$ & $3-\mathrm{Cl}$ & 0.5 & 0.5 & 0.25 & 1 & 0.5 & 0.5 & 0.25 & 0.25 & 0.25 & 4 \\
\hline $\mathbf{6}$ & $4-\mathrm{Cl}$ & 0.5 & 0.5 & 0.25 & 1 & 0.5 & 0 & 0 & 0 & 0.25 & 3 \\
\hline $\mathbf{7}$ & $2-\mathrm{OH}$ & 1 & 2 & 2 & 1 & 0 & 0 & 1 & 1 & 1 & 9 \\
\hline $\mathbf{8}$ & $4-\mathrm{OH}$ & 1 & 1 & 1 & 0.5 & 0.5 & 1 & 2 & 1 & 1 & 10 \\
\hline $\mathbf{9}$ & $4-\mathrm{OCH}_{3}$ & 1 & 0.5 & 0.5 & 1 & 1 & 0 & 1 & 1 & 1 & 9 \\
\hline $\mathbf{1 0}$ & $4-\mathrm{CH}_{3}$ & 0.5 & 1 & 0.5 & 2 & 2 & 0.5 & 0.5 & 1 & 1 & 7 \\
\hline $\mathbf{1 1}$ & $2-\mathrm{NO}_{2}$ & 2 & 3 & 3 & 1 & 1 & 1 & 0.5 & 1 & 0 & 12 \\
\hline $\mathbf{1 2}$ & $4-\mathrm{NO}_{2}$ & 2 & 3 & 3 & 1 & 0 & 0 & 0.5 & 1 & 0 & 7 \\
\hline
\end{tabular}

Number of leaf discs consumed by the insect (Values are mean $+\mathrm{SE}$ of five).

Table 9. Insect antifeedant activity of compound 6 1-(2-benzyloxy-(3-methoxyacrylo)-1-naphthyl)4-chlorophenyl chalcone at the concentration of 50,100 and $150 \mathrm{ppm}$.

\begin{tabular}{|l|l|l|l|l|l|l|l|l|l|l|}
\hline $\mathrm{ppm}$ & $\begin{array}{l}4-6 \\
\mathrm{pm}\end{array}$ & $\begin{array}{l}6-8 \\
\mathrm{pm}\end{array}$ & $\begin{array}{l}8-10 \\
\mathrm{pm}\end{array}$ & $\begin{array}{l}10-12 \\
\mathrm{pm}\end{array}$ & $\begin{array}{l}12-6 \\
\mathrm{am}\end{array}$ & $\begin{array}{l}6-8 \\
\mathrm{am}\end{array}$ & $\begin{array}{l}8 \mathrm{am}- \\
12 \mathrm{Nn}\end{array}$ & $\begin{array}{l}12 \mathrm{Nn}- \\
2 \mathrm{pm}\end{array}$ & $\begin{array}{l}2-4 \\
\mathrm{pm}\end{array}$ & $\begin{array}{l}\text { Total leaf disc } \\
\text { consumed in } 24 \mathrm{~h}\end{array}$ \\
\hline 50 & 0.5 & 0.5 & 0 & 0 & 0 & 0 & 0 & 0 & 0 & 0.1 \\
\hline 100 & 0.1 & 0.5 & 0 & 0 & 0 & 0 & 0 & 0 & 0 & 0.15 \\
\hline 150 & 0 & 0.25 & 0.25 & 0 & 0.0 & 0 & 0 & 0 & 0 & 0.05 \\
\hline
\end{tabular}

Number of leaf discs consumed by the insect (Values are mean + SE of five).

\section{CONCLUSIONS}

A series of 2-benzyloxy-(3-methoxyacrylate)-1-naphthyl enone derivatives have been synthesized by $\mathrm{LiOH}_{\mathrm{H}} \mathrm{O}$ catalyzed Claisen-Schmidt condensation of 2-benzyloxy-(3methoxyacrylate)-1-naphthyl ketone and benzaldehydes. The yields of the enones are more than $90 \%$. Synthesized enones were characterized by the physical constants and spectral data. The spectral group frequencies such as infrared $v \mathrm{CO}_{\text {s-cis, s-trans, }} \mathrm{CO}_{\text {ester, }}$ NMR chemical shifts $(\delta$, ppm $)$ of $\mathrm{H}_{\alpha}, \mathrm{H}_{\beta}, \mathrm{C}_{\alpha}, \mathrm{C}_{\beta}$, $\mathrm{CO}$ of enone moiety, $\mathrm{H}_{\beta^{\prime}}$ and $\mathrm{CO}$ of acrylate moieties have been correlated with Hammett substituent constants, $\mathrm{F}$ and $\mathrm{R}$ parameters. From the results of statistical analysis almost all correlations gave satisfactory correlation in single and multiple linear-regression analysis. of The antimicrobial activities of synthesized enones have been evaluated using Bauer-Kirby methods. Enone derivatives containing halogens, methoxy, methyl and nitro substituents have shown good antimicrobial activities. The antioxidant activities of the enones were measured by DPPH radical scavenging method. The compounds containing hydroxyl and methoxy substituents shown antioxidant activity. The insect antifeedant activities of ketones have been evaluated using Dethler's leaf-disc bioassay method. The ketone 6 1-(2-benzyloxy-(3-methoxyacrylate)-1-naphthyl)-4-chloro phenyl chalcone showed an appreciable antifeedant activity.

\section{Acknowledgment}

The authors thank DST-NMR facility, Department of Chemistry, Annamalai University, Annamalainagar-608002, for recording NMR spectra for all compounds. 


\section{References}

[1] Thirunarayanan, G.; Gopalakrishnan, M.; Vanangamudi, G. Spectrochim. Acta. 2007, 67(A), 1106-1112.

[2] Thirunarayanan, G. Indian J. Chem. 2007, 46B, 1511-1517.

[3] Pantoustier, N.; Moins, S.; Wautier, M.; Degée, P.; Dubois, P.Chem. Commun. 2003, 340341.

[4] Tang, X.; Liang, X.; Gao, L.; Fan, X.; Zhou, Q. (2010) J. Polymer Sci. 48A, 2564 - 2570.

[5] Thirunarayanan, G., Int. J. Sci. Res. Know. 2013, 1(12), 480-596.

[6] Thirunarayanan, G. Int. Lett. Chem. Phys. Astro. 2014, 4, 109-116.

[7] Sanghi, R.; Srivastava, M. M. Green Chemistry-Environment Friendly Alternatives, Narosa Publishers, NewDelhi-110 015, 2003.

[8] Patel, A. K.; Patel, R. J.; Patel, K. H.; Patel R. M. J. Chil. Chem. Soc. (2009) 54, 228-233.

[9] Aravinda Reddy, P.; Ramachandra Reddy, G.; Babul Reddy, A.; Subbarami Reddy, N. (2011) Adv. Polymer Sci. Tech. An Int. J. 2011, 1 (1), 1-9

[10] Russom, C. L.; Drummond, R. A.; Hoffman, A. D. Bull. Environ. Cont. Toxicol. 1988, 41, 589-596.

[11] Dillingham, E. O.; Lawrence, W. H.; Autian, J.; Schmalz, G. J. Biomed. Mater. Res. 1983, 17, 945-957.

[12] Fujisawa, S.; Kadoma, Y. Molecules. 2012, 13, 758-773.

[13] Dearfield, K. L.; Millis, C. S.; Harrington-Brock, K.; Doerr, C. L.; Moore, M. M. Mutagenesis. 1989, 4(5), 381-393.

[14] Vanangamudi, G.; Subramanian M.; Thirunarayanan, G.; Arabian J. Chem. 2013, DOI: 10.1016/j.arabjc.2013.03.006, 2013.

[15] Chen J. C.; Qian L.; Wu W. J.; Chen L. M.; Zheng K. C. J. Mol. Struct. (Theochem). 2005, 756(1-3), 167-172.

[16] Ahmad, A. L. M.; Dowsett, A. B.; Tyrrell, D. A. J. Antiviral Res. 1987, 8(1), 27-39.

[17] Carelli, V.; Di Colo, G.; Nannipieri, E.; Poli, B.; Serafini, M.F. Int. J. Pharm. 2000, 202( 1-2), 103-112.

[18] Méndez, J. A.; Fernández, M.; González-Corchón, A.; Salvado, M.; Collía, F.; De Pedro, J. A.; Levenfeld, B. L.; López-Bravo, A.; Vázquez, B.; San Román, J. Biomaterials. 2004, 25(12), 2381-2392.

[19] Arulkumaran, R.; Sundararajan, R.; Vanangamudi, G.; Subramanian, M.; Ravi, K.; Sathiyendiran, V.; Srinivasan, S.; Thirunarayanan, G. IUP. J. Chem. 2010, 3, 82-98.

[20] Lin, Y. M.; Zhon, Y.; Flavin, M. T.; Zhon, M.; Ne, W.; Chen, F. C. Bioorg. Med. Chem. 2002, 10, 2795-2802.

[21] Thirunarayanan, G.; Surya, S.; Srinivasan, S.; Vanangamudi, G.; Sathiyendiran, V. Spectrochim. Acta. 2010, 75(A), 152-156.

[22] Thirunarayanan, G. J. Indian Chem. Soc. 2008, 85, 447-451.

[23] Thirunarayanan, G.; Mayavel, P.; Thirumurthy, K. Spectrochim. Acta. 91A, 2012, 18-22.

[24] Ranganathan, K.; Arulkumaran, R.; Kamalakkannan, D.; Sundararajan, R.; Sakthinathan, S. P.; Vijayakumar, S.; Suresh, R.; Vanangamudi, G.; Thirumurthy, K.; Mayavel, P.; Thirunarayanan, G. Int. J. Pharm. Med. Biol. Sci. 2012, 1(1), 62-85. 
[25] Sundararajan, R.; Arulkumaran, R.; Vijayakumar, S.; Kamalakkannan, D.; Suresh, R.; Ranganathan, K.; Sakthinathan, S P.; Vanangamudi, G.; Thirumurthy, K.; Mayavel, P.; Thirunarayanan, G. Int. J. Pharm. Chem. Sci. 2012, 1(4), 1657-1677.

[26] Janaki, P.; Sekar, K. G.; Thirunarayanan, G. J. Saudi Chem. Soc. 2012. Doi.10.1016/j.jscs.2012.11.013

[27] Thirunarayanan, G. Vanangamudi, G.; Sathiyendiran, V.; Ravi, K. Indian J. Chem. 2011, 50B, 593-604,

[28. Sasikala, S.; Thirumurthy, K.; Mayavel, P.; Thirunarayanan, G. Org. Med. Chem. Lett. (2012) doi:10.1186/2191-2858-2-20.

[29] John Joseph, S.; Arulkumaran, R.; Kamalakkannan, D.; Sakthinathan, S. P.; Sundararajan, R.; Suresh, R.; Vijayakumar, S.; Ranganathan, K.; Kalyanasundaram, N.; Vanangamudi, G.; Thirunarayanan, G. Int. Lett. Chem. Phys. Astro. 2014, 4, 48-65.

[30] Thirunarayanan, G. Int. Lett. Chem. Phys. Astro. 2013, 9(2), 152- 161.

[31] Thirunarayanan, G.; Mayavel, P.; Thirumurthy, K.; Dineshkumar, S.; Sasikala, R.; Nisha, P.; Nithyaranjani, A. Euro. Chem. Bull. 2013, 2(9), 598-605.

[32] Sundararajan, R.; Arulkumaran, R.; Vijayakumar, S.; Kamalakkannan, D.; Suresh, R.; John Joseph, S.; Ranganathan, K.; Vanangamudi, G.; Subramanian, M.; Thirunarayanan, G.; Muthuvel, I.; Krishnakumar, B. Q-science Connect. (2013)

DOI: http://dx.doi.org/10.5339/connect.2013.30

[33] Swain, C. G.; Lupton Jr, E. C. J. Am. Chem. Soc. 1968, 90 (16), 4328-4337.

[34] Bauer, A. W.; Kirby, W. M. M.; Sherris, J. C.; Truck, M. Am. J. Clin. Pathol. 1966, 45, 493-498.

[35] Thirunarayanan, G.; Vanangamudi, G.; Subramanian, M. Org. Chem.: An Indian J. 2013, 9, 1-14.

[36] V. G. Dethler's Chemical Insect Attractants and Repellents, Blackistan, Philadelphia, 1947, p. 210 . 